\title{
Monodromy map for tropical Dolbeault cohomology
}

\author{
Yifeng Liu
}

\begin{abstract}
We define monodromy maps for the tropical Dolbeault cohomology of algebraic varieties over non-Archimedean fields. We propose a conjecture of Hodge isomorphisms via monodromy maps and provide some evidence.
\end{abstract}

\section{Introduction}

Let $K$ be a complete non-Archimedean field. For a separated scheme $X$ of finite type over $K$, one has the associated $K$-analytic space $X^{\text {an }}$ in the sense of Berkovich [Ber93]. Using the $\mathbb{Q}$-sheaf $\mathscr{T}_{X}^{p}$ an on $X^{\text {an }}$ constructed in [Liu17], we obtain a new cohomology theory $\mathrm{H}_{\text {trop }}^{p, q}(X)$, functorial in $X$. They are rational vector spaces satisfying $\mathrm{H}_{\text {trop }}^{p, q}(X) \neq 0$ only when $0 \leqslant p, q \leqslant \operatorname{dim} X$. Denote by $\mathrm{h}_{\text {trop }}^{p, q}(X)$ the dimension of $\mathrm{H}_{\text {trop }}^{p, q}(X)$, which might be infinity.

Using a bicomplex $\mathscr{A}_{X^{\bullet}}^{\bullet \bullet}$ of $\mathbb{R}$-sheaves of real forms on $X^{\text {an }}$, constructed by Chambert-Loir and Ducros [CD12], we can recover $\mathrm{H}_{\text {trop }}^{p, q}(X)_{\mathbb{R}}:=\mathrm{H}_{\text {trop }}^{p, q}(X) \otimes_{\mathbb{Q}} \mathbb{R}$ via certain Dolbeault cohomology groups. In this article, we consider a natural question, motivated by complex geometry, about the Hodge relation: Do we have the equality $\mathrm{h}_{\text {trop }}^{p, q}(X)=\mathrm{h}_{\text {trop }}^{q, p}(X)$ ?

However, the perspective we take is very different from the one in complex geometry. In fact, we will construct, for $p \geqslant 1$ and $q \geqslant 0$, a functorial map

$$
\mathrm{N}_{X}: \mathrm{H}_{\text {trop }}^{p, q}(X)_{\mathbb{R}} \rightarrow \mathrm{H}_{\text {trop }}^{p-1, q+1}(X)_{\mathbb{R}},
$$

which we call the monodromy map, via constructing a canonical map $\mathscr{A}_{X^{\text {an }}}^{p, q} \rightarrow \mathscr{A}_{X^{\text {an }}}^{p-1, q+1}$ on the level of sheaves. Such a map does not exist in complex geometry. We conjecture (see Conjecture 5.3) that over certain algebraically closed complete non-Archimedean fields $K$, the iterated map

$$
\mathrm{N}_{X}^{p-q}: \mathrm{H}_{\text {trop }}^{p, q}(X)_{\mathbb{R}} \rightarrow \mathrm{H}_{\text {trop }}^{q, p}(X)_{\mathbb{R}}
$$

is an isomorphism for $p \geqslant q$ if $X$ is proper smooth. We view such an isomorphism as the Hodge isomorphism for tropical Dolbeault cohomology. If this holds, then we have the following numerical relation for the corresponding Hodge numbers:

$$
\mathrm{h}_{\text {trop }}^{p, 0}(X) \leqslant \mathrm{h}_{\text {trop }}^{p-1,1}(X) \leqslant \cdots \cdots \mathrm{h}_{\text {trop }}^{1, p-1}(X) \geqslant \mathrm{h}_{\text {trop }}^{0, p}(X) .
$$

This is apparently new from the results in complex geometry. In fact, we believe that the Hodge isomorphism and the above numerical relation should have a connection with the weight spectral

Received 7 July 2017, accepted in final form 25 January 2018.

2010 Mathematics Subject Classification 14G22.

Keywords: tropical Dolbeault cohomology, monodromy map.

This journal is (C) Foundation Compositio Mathematica 2019. This article is distributed with Open Access under the terms of the Creative Commons Attribution Non-Commercial License, which permits non-commercial reuse, distribution, and reproduction in any medium, provided that the original work is properly cited. For commercial re-use, please contact the Foundation Compositio Mathematica.

The author is partially supported by NSF grant DMS-1702019 and a Sloan Research Fellowship. 


\section{Monodromy map for tropical Dolbeault COHOMOlogy}

sequence. We note that the map $\mathrm{N}_{X}^{p}: \mathrm{H}_{\text {trop }}^{p, 0}(X)_{\mathbb{R}} \rightarrow \mathrm{H}_{\text {trop }}^{0, p}(X)_{\mathbb{R}}$ is simply induced by the "flipping" map J, see [CD12], multiplied by $p$ !; see Lemma 3.2(1).

The theorem below provides certain evidence toward our expected Hodge isomorphism. The proof uses arithmetic geometry, especially the weight spectral sequence as we have just mentioned. The method is restrictive in the sense that any further extension would seem to rely on the weight-monodromy conjecture (Conjecture 4.5) in the mixed-characteristic case, among other difficulties. Therefore, it is very interesting and important to find a somewhat analytic proof of the results here. However, the situation is rather subtle since we know that for some algebraically closed, complete, non-Archimedean fields $K$, such as the field of Puiseux series $\mathbb{C}\{\{t\}\}$, the map $\mathrm{N}_{X}: \mathrm{H}_{\text {trop }}^{1,0}(X)_{\mathbb{R}} \rightarrow \mathrm{H}_{\text {trop }}^{0,1}(X)_{\mathbb{R}}$ may fail to be an isomorphism (see Remark 5.4).

In this article, by a non-Archimedean field, we mean a complete topological field equipped with a nontrivial, non-Archimedean valuation of rank one. For a non-Archimedean field $K$, we denote the ring of integers by $K^{\circ}$, the residue field by $\widetilde{K}$, and a fixed algebraic closure by $K^{\mathrm{a}}$, with $\widehat{K^{\mathrm{a}}}$ its completion.

Theorem 1.1. Let $X_{0}$ be a proper, smooth scheme over a non-Archimedean field $K_{0}$. Let $K$ be a closed subfield of $\widehat{K_{0}^{\mathrm{a}}}$ containing $K_{0}$. Put $X:=X_{0} \otimes_{K_{0}} K$.

(i) (Corollary 5.12) Suppose that $K_{0}$ is isomorphic to $k((t))$ for $k$ either a finite field or a field of characteristic zero. Then the monodromy map

$$
\mathrm{N}_{X}^{p}: \mathrm{H}_{\text {trop }}^{p, 0}(X)_{\mathbb{R}} \rightarrow \mathrm{H}_{\text {trop }}^{0, p}(X)_{\mathbb{R}}
$$

is injective for every $p \geqslant 0$. In particular, $\mathrm{h}_{\mathrm{trop}}^{p, 0}(X)$ is finite.

(ii) (Theorem 5.13) Suppose that $K_{0}$ is either a finite extension of $\mathbb{Q}_{p}$ or isomorphic to $k((t))$ for $k$ a finite field, $K=\widehat{K_{0}^{\mathrm{a}}}$, and $X_{0}$ admits a proper, strictly semistable model over $K_{0}^{\circ}$. Then the monodromy map

$$
\mathrm{N}_{X}: \mathrm{H}_{\text {trop }}^{1,0}(X)_{\mathbb{R}} \rightarrow \mathrm{H}_{\text {trop }}^{0,1}(X)_{\mathbb{R}}
$$

is an isomorphism.

Remark 1.2. Let $K$ be an algebraically closed, non-Archimedean field.

(1) In his thesis, Jell proved that for a proper, smooth scheme $X$ over $K$ of dimension $n$, the map $\mathrm{N}_{X}^{p}: \mathrm{H}_{\text {trop }}^{p, 0}(X)_{\mathbb{R}} \rightarrow \mathrm{H}_{\text {trop }}^{0, p}(X)_{\mathbb{R}}$ is injective for $p=0,1, n$ [Jel16, Proposition 3.4.11].

(2) In [JW18], Jell and Wanner proved that for $X$ either $\mathbf{P}_{K}^{1}$ or a (proper, smooth) Mumford curve over $K$, the map $\mathrm{N}_{X}: \mathrm{H}_{\text {trop }}^{1,0}(X)_{\mathbb{R}} \rightarrow \mathrm{H}_{\text {trop }}^{0,1}(X)_{\mathbb{R}}$ is an isomorphism.

Their methods in both papers are analytic.

In [MZ14], Mikhalkin and Zharkov study the tropical homology groups $\mathrm{H}_{p, q}(X)$ of a tropical space $X$. Assuming $X$ compact, they define a map

$$
\phi \cap: \mathrm{H}_{p, q}(X)_{\mathbb{R}} \rightarrow \mathrm{H}_{p+1, q-1}(X)_{\mathbb{R}}
$$

using their eigenwave $\phi$. In fact, as shown in [JSS18], one can compute the tropical homology $\mathrm{H}_{p, q}(X)_{\mathbb{R}}$, or rather the tropical cohomology, via superforms in [CD12] as well. Then our construction in Section 2 would give rise to a map $\mathrm{N}_{X}: \mathrm{H}_{p, q}(X)_{\mathbb{R}} \rightarrow \mathrm{H}_{p+1, q-1}(X)_{\mathbb{R}}$ (or rather on cohomology) for any tropical space $X$. We expect that $\mathrm{N}_{X}$ and $\phi \cap$ should coincide when $X$ is compact, possibly up to an elementary factor.

Moreover, in [MZ14], the authors prove that when $X$ is a realizable, smooth, compact tropical space, the iterated map $\phi^{q-p} \cap: \mathrm{H}_{p, q}(X)_{\mathbb{R}} \rightarrow \mathrm{H}_{q, p}(X)_{\mathbb{R}}$ is an isomorphism. They first realize $X$ as 
the tropical limit of a complex, projective, one-parameter, semistable degeneration $\mathcal{X}$ such that all strata of the singular fiber $\mathcal{X}_{0}$ are blow-ups of projective spaces. By the work [IKMZ16], one knows that $\mathrm{H}_{p, q}(X)_{\mathbb{Q}}$ can be identified with $\mathrm{E}_{2}$-terms of the Steenbrink-Illusie spectral sequence associated with $\mathcal{X}$. Moreover, Mikhalkin and Zharkov show that under such an identification, the map $\phi \cap$ is simply the monodromy map on $\mathrm{E}_{2}$-terms. In our work, $X$ is a proper, smooth $^{1}$ scheme, and we relate our tropical Dolbeault cohomology $\mathrm{H}_{\text {trop }}^{p, q}(X)$ to $\mathrm{E}_{2}$-terms of the weight spectral sequence of (various) semistable alterations $\mathcal{X}$ of $X$ (see Definition 5.5 for the precise meaning) for certain $p$ and $q$, under which $\mathrm{N}_{X}$ is essentially the monodromy map. In our setup, the semistable scheme $\mathcal{X}$ is very general, so that its $\mathrm{E}_{2}$-terms cannot be read off solely from the dual complex of $\mathcal{X}$; hence, we do not obtain a strict identification for general $p$ and $q$. Nevertheless, it would be interesting to compare our approach relating to the weight spectral sequence and their approach relating to the Steenbrink-Illusie spectral sequence in [MZ14, IKMZ16].

\section{Conventions}

Let $K$ be a non-Archimedean field. All $K$-analytic (Berkovich) spaces are assumed to be good [Ber93, Remark 1.2.16], Hausdorff, and strictly K-analytic [Ber93, Proposition 1.2.15].

For a $K$-analytic space $X$, we use $\mathrm{H}^{\bullet}(X,-)$ to indicate the cohomology group with respect to the underlying topology of $X$, and we use $\mathrm{H}_{\text {ét }}^{\bullet}(X,-)$ to indicate the cohomology group with respect to the étale topology of $X$.

\section{Monodromy maps for superforms on vector spaces}

In this section, we review the construction of superforms on vector spaces from [CD12] and introduce the corresponding monodromy map.

Let $V$ be an $\mathbb{R}$-vector space of dimension $n$. Let $\mathrm{T}_{V}$ be the tangent space of $V$ and $\mathrm{T}_{V}^{*}$ its dual space. For every open subset $U$ of $V$ and integers $p, q \geqslant 0$, we have the space of $(p, q)$-forms on $U$,

$$
\mathscr{A}_{V}^{p, q}(U):=\mathscr{A}_{V}(U) \otimes_{\mathbb{R}} \bigwedge^{p} \mathrm{~T}_{V}^{*} \otimes_{\mathbb{R}} \bigwedge^{q} \mathrm{~T}_{V}^{*}
$$

where $\mathscr{A}_{V}(U)$ is the $\mathbb{R}$-algebra of smooth functions on $U$. The direct sum

$$
\mathscr{A}_{V}^{*, *}(U):=\bigoplus \mathscr{A}_{V}^{p, q}(U)
$$

forms a bigraded $\mathbb{R}$-algebra with the following commutativity law: if $\omega$ is a $(p, q)$-form and $\omega^{\prime}$ is a $\left(p^{\prime}, q^{\prime}\right)$-form, then we have

$$
\omega^{\prime} \wedge \omega=(-1)^{(p+q)\left(p^{\prime}+q^{\prime}\right)} \omega \wedge \omega^{\prime} .
$$

Moreover, $\wedge: \mathscr{A}_{V}^{p, 0}(U) \times \mathscr{A}_{V}^{0, q}(U) \rightarrow \mathscr{A}_{V}^{p, q}(U)$ is simply the map induced by the tensor product map.

The real vector spaces $\mathscr{A}_{V}^{\bullet \bullet \bullet}(U)$ form a bicomplex with two differentials

$$
\mathrm{d}^{\prime}: \mathscr{A}_{V}^{p, q}(U) \rightarrow \mathscr{A}_{V}^{p+1, q}(U), \quad \mathrm{d}^{\prime \prime}: \mathscr{A}_{V}^{p, q}(U) \rightarrow \mathscr{A}_{V}^{p, q+1}(U) .
$$

We also have a convolution map $\mathrm{J}: \mathscr{A}_{V}^{p, q}(U) \rightarrow \mathscr{A}_{V}^{q, p}(U)$.

\footnotetext{
${ }^{1}$ It is worth noting that the tropicalization of a smooth analytic space, such as $X^{\text {an }}$, under a local tropical chart is in general not a smooth tropical space.
} 


\section{MONODROMY MAP FOR TROPICAL DOLBEAUlT COHOMOLOGY}

In terms of coordinates, they are described as follows. Let $\left\{x_{1}, \ldots, x_{n}\right\}$ be a system of coordinates of $V$. Then a $(p, q)$-form can be written as

$$
\omega=\sum_{|I|=p,|J|=q} \omega_{I, J}(x) \mathrm{d}^{\prime} x_{I} \otimes \mathrm{d}^{\prime \prime} x_{J}=\sum_{|I|=p,|J|=q} \omega_{I, J}(x) \mathrm{d}^{\prime} x_{I} \wedge \mathrm{d}^{\prime \prime} x_{J},
$$

where $I$ and $J$ are subsets of $\{1, \ldots, n\}$ and the $\omega_{I, J}(x)$ are smooth functions on $U$. For such a form $\omega$, we have

$$
\begin{aligned}
\mathrm{d}^{\prime} \omega & =\sum_{|I|=p,|J|=q} \sum_{i=1}^{n} \frac{\partial \omega_{I, J}(x)}{\partial x_{i}} \mathrm{~d}^{\prime} x_{i} \wedge \mathrm{d}^{\prime} x_{I} \wedge \mathrm{d}^{\prime \prime} x_{J}, \\
\mathrm{~d}^{\prime \prime} \omega & =(-1)^{p} \sum_{|I|=p,|J|=q} \sum_{j=1}^{n} \frac{\partial \omega_{I, J}(x)}{\partial x_{j}} \mathrm{~d}^{\prime} x_{I} \wedge \mathrm{d}^{\prime \prime} x_{j} \wedge \mathrm{d}^{\prime \prime} x_{J}, \\
\mathrm{~J} \omega & =(-1)^{p q} \sum_{|I|=p,|J|=q} \omega_{I, J}(x) \mathrm{d}^{\prime} x_{J} \wedge \mathrm{d}^{\prime \prime} x_{I} .
\end{aligned}
$$

We remind readers of the following relations from [CD12, Section 1.2]:

$$
\mathrm{d}^{\prime \prime}=\mathrm{Jd}^{\prime} \mathrm{J}, \quad \mathrm{Jd}^{\prime}=\mathrm{d}^{\prime \prime} \mathrm{J}, \quad \mathrm{Jd}^{\prime \prime}=\mathrm{d}^{\prime} \mathrm{J}, \quad \mathrm{d}^{\prime}=\mathrm{Jd}^{\prime \prime} \mathrm{J},
$$

and for a $(p, q)$-form $\omega$ and a $\left(p^{\prime}, q^{\prime}\right)$-form $\omega^{\prime}$,

$$
\begin{aligned}
\mathrm{d}^{\prime}\left(\omega \wedge \omega^{\prime}\right) & =\mathrm{d}^{\prime} \omega \wedge \omega^{\prime}+(-1)^{p+q} \omega \wedge \mathrm{d}^{\prime} \omega^{\prime} \\
\mathrm{d}^{\prime \prime}\left(\omega \wedge \omega^{\prime}\right) & =\mathrm{d}^{\prime \prime} \omega \wedge \omega^{\prime}+(-1)^{p+q} \omega \wedge \mathrm{d}^{\prime \prime} \omega^{\prime}
\end{aligned}
$$

Now we are going to define a map $\mathrm{N}: \mathscr{A}_{V}^{p, q}(U) \rightarrow \mathscr{A}_{V}^{p-1, q+1}(U)$ for $p \geqslant 1$. We first recall the notion of coevaluation map. Let $W$ be an arbitrary finite-dimensional $\mathbb{R}$-vector space with $W^{*}$ its dual space. We have a canonical evaluation map

$$
\mathrm{ev}: W^{*} \otimes_{\mathbb{R}} W \rightarrow \mathbb{R} .
$$

We define the coevaluation map to be the unique linear map

$$
\operatorname{coev}: \mathbb{R} \rightarrow W \otimes_{\mathbb{R}} W^{*}
$$

such that both composite maps

$$
\begin{gathered}
W^{*} \stackrel{1_{W^{*} \otimes \text { coev }}^{\longrightarrow}}{\longrightarrow} W^{*} \otimes_{\mathbb{R}}\left(W \otimes_{\mathbb{R}} W^{*}\right) \stackrel{\sim}{\rightarrow}\left(W^{*} \otimes_{\mathbb{R}} W\right) \otimes_{\mathbb{R}} W^{*} \stackrel{\mathrm{ev} \otimes 1_{W^{*}}}{\longrightarrow} W^{*}, \\
W \stackrel{\operatorname{coev} \otimes 1_{W}}{\longrightarrow}\left(W \otimes_{\mathbb{R}} W^{*}\right) \otimes_{\mathbb{R}} W \stackrel{\sim}{\rightarrow} W \otimes_{\mathbb{R}}\left(W^{*} \otimes_{\mathbb{R}} W\right) \stackrel{1_{W} \otimes \mathrm{ev}}{\longrightarrow} W
\end{gathered}
$$

are identity maps. In fact, for an arbitrary basis $\left\{w_{1}, \ldots, w_{n}\right\}$ of $W$, with the dual basis $\left\{w_{1}^{*}, \ldots\right.$, $\left.w_{n}^{*}\right\}$ of $W^{*}$, the map coev sends 1 to $\sum_{i=1}^{n} w_{i} \otimes w_{i}^{*}$.

Now we apply the coevaluation map to the vector space $W=\mathrm{T}_{V}$.

Definition 2.1. Let $p \geqslant 1$ be an integer. Define the map

$$
\mathrm{N}: \mathscr{A}_{V}^{p, q}(U) \rightarrow \mathscr{A}_{V}^{p-1, q+1}(U)
$$


to be the composite map

$$
\begin{aligned}
& \mathscr{C}^{\infty}(U) \otimes_{\mathbb{R}} \bigwedge^{p} \mathrm{~T}_{V}^{*} \otimes_{\mathbb{R}} \bigwedge^{q} \mathrm{~T}_{V}^{*} \stackrel{\sim}{\rightarrow} \mathscr{C}^{\infty}(U) \otimes_{\mathbb{R}} \bigwedge^{p} \mathrm{~T}_{V}^{*} \otimes_{\mathbb{R}} \mathbb{R} \otimes_{\mathbb{R}} \bigwedge^{q} \mathrm{~T}_{V}^{*} \\
& \rightarrow \mathscr{C}^{\infty}(U) \otimes_{\mathbb{R}} \bigwedge^{p} \mathrm{~T}_{V}^{*} \otimes_{\mathbb{R}}\left(\mathrm{T}_{V} \otimes_{\mathbb{R}} \mathrm{T}_{V}^{*}\right) \otimes_{\mathbb{R}} \bigwedge^{q} \mathrm{~T}_{V}^{*} \\
& \stackrel{\sim}{\rightarrow} \mathscr{C}^{\infty}(U) \otimes_{\mathbb{R}}\left(\bigwedge^{p} \mathrm{~T}_{V}^{*} \otimes_{\mathbb{R}} \mathrm{T}_{V}\right) \otimes_{\mathbb{R}}\left(\mathrm{T}_{V}^{*} \otimes_{\mathbb{R}} \bigwedge^{q} \mathrm{~T}_{V}^{*}\right) \\
& \rightarrow \mathscr{C}^{\infty}(U) \otimes_{\mathbb{R}}^{p-1} \bigwedge^{\mathrm{T}_{V}^{*}} \otimes_{\mathbb{R}} \bigwedge^{q+1} \mathrm{~T}_{V}^{*},
\end{aligned}
$$

where the second map is given by the coevaluation map for $\mathrm{T}_{V}$ and the last map is given by the contraction map and the wedge product. For $0 \leqslant r \leqslant p$, we denote by

$$
\mathrm{N}^{r}: \mathscr{A}_{V}^{p, q}(U) \rightarrow \mathscr{A}_{V}^{p-r, q+r}(U)
$$

the consecutive composition.

In terms of the coordinates $\left\{x_{1}, \ldots, x_{n}\right\}$ of $V$, for

$$
\omega=\sum_{I=\left\{i_{1}<\cdots<i_{p}\right\}, J=\left\{j_{1}<\cdots<j_{q}\right\}} \omega_{I, J}(x) \mathrm{d}^{\prime} x_{i_{1}} \wedge \cdots \wedge \mathrm{d}^{\prime} x_{i_{p}} \wedge \mathrm{d}^{\prime \prime} x_{j_{1}} \wedge \cdots \wedge \mathrm{d}^{\prime \prime} x_{j_{q}}
$$

with $p \geqslant 1$, we have

$$
\begin{aligned}
\mathrm{N} \omega & =\sum_{k=1}^{p} \sum_{I, J}(-1)^{p-k} \omega_{I, J}(x) \mathrm{d}^{\prime} x_{i_{1}} \wedge \cdots \wedge \widehat{\mathrm{d}^{\prime} x_{i_{k}}} \wedge \cdots \wedge \mathrm{d}^{\prime} x_{i_{p}} \wedge \mathrm{d}^{\prime \prime} x_{i_{k}} \wedge \mathrm{d}^{\prime \prime} x_{j_{1}} \wedge \cdots \wedge \mathrm{d}^{\prime \prime} x_{j_{q}} \\
& =\sum_{k=1}^{p} \sum_{I, J}(-1)^{p-k} \omega_{I, J}(x) \mathrm{d}^{\prime} x_{I \backslash\left\{i_{k}\right\}} \wedge \mathrm{d}^{\prime \prime} x_{i_{k}} \wedge \mathrm{d}^{\prime \prime} x_{J} .
\end{aligned}
$$

Lemma 2.2. We have $\mathrm{Nd}^{\prime \prime}=\mathrm{d}^{\prime \prime} \mathrm{N}: \mathscr{A}_{V}^{p, q}(U) \rightarrow \mathscr{A}_{V}^{p-1, q+2}(U)$ for $p \geqslant 1$.

Proof. Take a $(p, q)$-form $\omega$ as in (2.1). We have

$$
\begin{aligned}
\mathrm{d}^{\prime \prime} \mathrm{N} \omega & =\mathrm{d}^{\prime \prime} \sum_{k=1}^{p} \sum_{I, J}(-1)^{p-k} \omega_{I, J}(x) \mathrm{d}^{\prime} x_{I \backslash\left\{i_{k}\right\}} \wedge \mathrm{d}^{\prime \prime} x_{i_{k}} \wedge \mathrm{d}^{\prime \prime} x_{J} \\
& =-\sum_{I, J} \sum_{j=1}^{n} \sum_{k=1}^{p} \frac{(-1)^{k} \partial \omega_{I, J}(x)}{\partial x_{j}} \mathrm{~d}^{\prime} x_{I \backslash\left\{i_{k}\right\}} \wedge \mathrm{d}^{\prime \prime} x_{j} \wedge \mathrm{d}^{\prime \prime} x_{i_{k}} \wedge \mathrm{d}^{\prime \prime} x_{J}
\end{aligned}
$$

On the other hand, we have

$$
\begin{aligned}
\mathrm{Nd}^{\prime \prime} \omega & =\mathrm{N}(-1)^{p} \sum_{I, J} \sum_{j=1}^{n} \frac{\partial \omega_{I, J}(x)}{\partial x_{j}} \mathrm{~d}^{\prime} x_{I} \wedge \mathrm{d}^{\prime \prime} x_{j} \wedge \mathrm{d}^{\prime \prime} x_{J} \\
& =\sum_{k=1}^{p} \sum_{I, J} \sum_{j=1}^{n} \frac{(-1)^{k} \partial \omega_{I, J}(x)}{\partial x_{j}} \mathrm{~d}^{\prime} x_{I \backslash\left\{i_{k}\right\}} \wedge \mathrm{d}^{\prime \prime} x_{i_{k}} \wedge \mathrm{d}^{\prime \prime} x_{j} \wedge \mathrm{d}^{\prime \prime} x_{J} \\
& =-\sum_{I, J} \sum_{j=1}^{n} \sum_{k=1}^{p} \frac{(-1)^{k} \partial \omega_{I, J}(x)}{\partial x_{j}} \mathrm{~d}^{\prime} x_{I \backslash\left\{i_{k}\right\}} \wedge \mathrm{d}^{\prime \prime} x_{j} \wedge \mathrm{d}^{\prime \prime} x_{i_{k}} \wedge \mathrm{d}^{\prime \prime} x_{J}
\end{aligned}
$$

The lemma follows. 


\section{MONODROMY MAP FOR TROPICAL DOLBEAUlT COHOMOLOGY}

Lemma 2.3. For a $(p, q)$-form $\omega$ and a $\left(p^{\prime}, q^{\prime}\right)$-form $\omega^{\prime}$ with $p, p^{\prime} \geqslant 1$ and $p+p^{\prime} \geqslant n+1$, we have $\mathrm{N} \omega \wedge \omega^{\prime}=-\omega \wedge \mathrm{N} \omega^{\prime}$.

Proof. By linearity, we may assume

$$
\omega=\omega(x) \mathrm{d}^{\prime} x_{I} \wedge \mathrm{d}^{\prime \prime} x_{J}, \quad \omega^{\prime}=\omega^{\prime}(x) \mathrm{d}^{\prime} x_{I^{\prime}} \wedge \mathrm{d}^{\prime \prime} x_{J^{\prime}} .
$$

If $\left|I \cap I^{\prime}\right| \geqslant 2$, then it is easy to see that $\mathrm{N} \omega \wedge \omega^{\prime}=\omega \wedge \mathrm{N} \omega^{\prime}=0$. Otherwise, $\left|I \cap I^{\prime}\right|=1$. Without loss of generality, we may assume $I \cap I^{\prime}=\{n\}$, hence $\mathrm{d}^{\prime} x_{I}=\mathrm{d}^{\prime} x_{I \backslash\{n\}} \wedge \mathrm{d}^{\prime} x_{n}$ and $\mathrm{d}^{\prime} x_{I^{\prime}}=\mathrm{d}^{\prime} x_{I^{\prime} \backslash\{n\}} \wedge \mathrm{d}^{\prime} x_{n}$. Then we have

$$
\begin{aligned}
\mathrm{N} \omega \wedge \omega^{\prime} & =\left(\omega(x) \mathrm{d}^{\prime} x_{I \backslash\{n\}} \wedge \mathrm{d}^{\prime \prime} x_{n} \wedge \mathrm{d}^{\prime \prime} x_{J}\right) \wedge\left(\omega^{\prime}(x) \mathrm{d}^{\prime} x_{I^{\prime}} \wedge \mathrm{d}^{\prime \prime} x_{J^{\prime}}\right) \\
& =(-1)^{p^{\prime}(q+1)} \omega(x) \omega^{\prime}(x) \mathrm{d}^{\prime} x_{I \backslash\{n\}} \wedge \mathrm{d}^{\prime} x_{I^{\prime}} \wedge \mathrm{d}^{\prime \prime} x_{n} \wedge \mathrm{d}^{\prime \prime} x_{J} \wedge \mathrm{d}^{\prime \prime} x_{J^{\prime}} \\
& =(-1)^{p^{\prime}(q+1)} \omega(x) \omega^{\prime}(x) \mathrm{d}^{\prime} x_{I \backslash\{n\}} \wedge \mathrm{d}^{\prime} x_{I^{\prime} \backslash\{n\}} \wedge \mathrm{d}^{\prime} x_{n} \wedge \mathrm{d}^{\prime \prime} x_{n} \wedge \mathrm{d}^{\prime \prime} x_{J} \wedge \mathrm{d}^{\prime \prime} x_{J^{\prime}}
\end{aligned}
$$

and

$$
\begin{aligned}
\omega \wedge \mathrm{N} \omega^{\prime} & =\left(\omega(x) \mathrm{d}^{\prime} x_{I} \wedge \mathrm{d}^{\prime \prime} x_{J}\right) \wedge\left(\omega^{\prime}(x) \mathrm{d}^{\prime} x_{I^{\prime} \backslash\{n\}} \wedge \mathrm{d}^{\prime \prime} x_{n} \wedge \mathrm{d}^{\prime \prime} x_{J^{\prime}}\right) \\
& =(-1)^{p^{\prime} q} \omega(x) \omega^{\prime}(x) \mathrm{d}^{\prime} x_{I} \wedge \mathrm{d}^{\prime} x_{I^{\prime} \backslash\{n\}} \wedge \mathrm{d}^{\prime \prime} x_{n} \wedge \mathrm{d}^{\prime \prime} x_{J} \wedge \mathrm{d}^{\prime \prime} x_{J^{\prime}} \\
& =(-1)^{p^{\prime} q} \omega(x) \omega^{\prime}(x) \mathrm{d}^{\prime} x_{I \backslash\{n\}} \wedge \mathrm{d}^{\prime} x_{n} \wedge \mathrm{d}^{\prime} x_{I^{\prime} \backslash\{n\}} \wedge \mathrm{d}^{\prime \prime} x_{n} \wedge \mathrm{d}^{\prime \prime} x_{J} \wedge \mathrm{d}^{\prime \prime} x_{J^{\prime}} \\
& =(-1)^{p^{\prime} q+\left(p^{\prime}-1\right)} \omega(x) \omega^{\prime}(x) \mathrm{d}^{\prime} x_{I \backslash\{n\}} \wedge \mathrm{d}^{\prime} x_{I^{\prime} \backslash\{n\}} \wedge \mathrm{d}^{\prime} x_{n} \wedge \mathrm{d}^{\prime \prime} x_{n} \wedge \mathrm{d}^{\prime \prime} x_{J} \wedge \mathrm{d}^{\prime \prime} x_{J^{\prime}} .
\end{aligned}
$$

The lemma follows.

Lemma 2.4. Let $V^{\prime}$ be another $\mathbb{R}$-vector space of dimension $n^{\prime}$ and $U^{\prime} \subset V^{\prime}$ an open subset. Let $\varphi: V^{\prime} \rightarrow V$ be an affine map such that $\varphi\left(U^{\prime}\right) \subset U$. Then for $p \geqslant 1$, we have

$$
\mathrm{N}^{\prime} \varphi^{*}=\varphi^{*} \mathrm{~N}: \mathscr{A}_{V}^{p, q}(U) \rightarrow \mathscr{A}_{V^{\prime}}^{p-1, q+1}\left(U^{\prime}\right),
$$

where $\mathrm{N}^{\prime}$ denotes the monodromy map for $V^{\prime}$.

Proof. We may assume that $\varphi$ is a linear map. It suffices to consider cases where $\varphi$ is injective or surjective.

Suppose that $\varphi$ is injective. Regard $V^{\prime}$ as a subspace of $V$. Choose a basis $\left\{x_{1}, \ldots, x_{n}\right\}$ of $V$ such that $V^{\prime}$ is spanned by $\left\{x_{1}, \ldots, x_{n^{\prime}}\right\}$ (with $n^{\prime} \leqslant n$ ). Take $\omega=\omega(x) \mathrm{d}^{\prime} x_{I} \wedge \mathrm{d}^{\prime \prime} x_{J} \in \mathscr{A}_{V}^{p, q}(U)$ for $I=\left\{i_{1}<\cdots<i_{p}\right\}$. If $i_{p}>n^{\prime}$, then $\mathrm{N}^{\prime} \varphi^{*} \omega=\varphi^{*} \mathrm{~N} \omega=0$. If $i_{p} \leqslant n^{\prime}$, then we have

$$
\mathrm{N}^{\prime} \varphi^{*} \omega=\varphi^{*} \mathrm{~N} \omega=\left.\left(\omega(x) \sum_{k=1}^{p}(-1)^{p-k} \mathrm{~d}^{\prime} x_{I \backslash\left\{i_{k}\right\}} \wedge \mathrm{d}^{\prime \prime} x_{i_{k}} \wedge \mathrm{d}^{\prime \prime} x_{J}\right)\right|_{U^{\prime}} .
$$

Suppose that $\varphi$ is surjective. Choose a basis $\left\{x_{1}, \ldots, x_{n^{\prime}}\right\}$ of $V^{\prime}$ such that $\operatorname{ker} \varphi$ is spanned by $\left\{x_{n+1}, \ldots, x_{n^{\prime}}\right\}$ (with $n \leqslant n^{\prime}$ ). We identify $V$ with the subspace of $V^{\prime}$ spanned by $\left\{x_{1}, \ldots, x_{n}\right\}$. Then, again, we have

$$
\mathrm{N}^{\prime} \varphi^{*} \omega=\varphi^{*} \mathrm{~N} \omega=\left.\left(\omega(\varphi(x)) \sum_{k=1}^{p}(-1)^{p-k} \mathrm{~d}^{\prime} x_{I \backslash\left\{i_{k}\right\}} \wedge \mathrm{d}^{\prime \prime} x_{i_{k}} \wedge \mathrm{d}^{\prime \prime} x_{J}\right)\right|_{U^{\prime}} .
$$

The lemma follows.

Lemma 2.5. The map $\mathrm{N}^{p}: \mathscr{A}_{V}^{p, 0}(U) \rightarrow \mathscr{A}_{V}^{0, p}(U)$ coincides with $p ! \cdot \mathrm{J}: \mathscr{A}_{V}^{p, 0}(U) \rightarrow \mathscr{A}_{V}^{0, p}(U)$.

Proof. This is elementary. 
Remark 2.6. The presheaf $U \mapsto \mathscr{A}_{V}^{p, q}(U)$ is already a sheaf on $V$. The maps $\mathrm{d}^{\prime}, \mathrm{d}^{\prime \prime}, \mathrm{J}, \wedge, \mathrm{N}$ induce maps of sheaves with the same relations. In particular, we have the map

$$
\mathrm{N}_{V}: \mathscr{A}_{V}^{p, q} \rightarrow \mathscr{A}_{V}^{p-1, q+1}
$$

of sheaves for $p \geqslant 1$ and the lemmas corresponding to Lemmas 2.2 and 2.3. Lemma 2.4 induces the equality $\left(\varphi_{*} \mathrm{~N}_{V^{\prime}}\right) \varphi^{*}=\varphi^{*} \mathrm{~N}_{V}: \mathscr{A}_{V}^{p, q} \rightarrow \varphi_{*} \mathscr{A}_{V^{\prime}}^{p-1, q+1}$ on the level of sheaves.

Let $P$ be a polyhedral complex ${ }^{2}$ in $V$ and $j: P \rightarrow V$ the tautological inclusion map. For every open subset $U$ of $P$, let $\mathscr{N}_{P}^{p, q}(U)$ be the subspace of $\left(j^{-1} \mathscr{A}_{V}^{p, q}\right)(U)$ of $(p, q)$-forms $\omega$ such that for every polyhedron $C$ of $P$, the restriction of $\omega$ to $\langle C\rangle$ is zero on $\langle C\rangle \cap U$, where $\langle C\rangle$ is the affine subset of $V$ spanned by $C$. It is clear that $\mathscr{N}_{P}^{p, q}$ is an $\mathbb{R}$-subsheaf of $j^{-1} \mathscr{A}_{V}^{p, q}$. Let $\mathscr{A}_{P}^{p, q}$ be the quotient sheaf $j^{-1} \mathscr{A}_{V}^{p, q} / \mathscr{N}_{P}^{p, q}$.

By Lemma 2.4, the monodromy map $j^{-1} \mathrm{~N}_{V}: j^{-1} \mathscr{A}_{V}^{p, q} \rightarrow j^{-1} \mathscr{A}_{V}^{p, q}$ preserves $\mathscr{N}_{P}^{p, q}$. Thus, we have an induced map

$$
\mathrm{N}_{P}: \mathscr{A}_{P}^{p, q} \rightarrow \mathscr{A}_{P}^{p-1, q+1}
$$

of sheaves for $p \geqslant 1$.

\section{Monodromy maps for real forms on analytic spaces}

In this section, we review the construction of $(p, q)$-forms in [CD12] and tropical Dolbeault cohomology, and we introduce the monodromy map on analytic spaces.

We fix a non-Archimedean field $K$. Let $X$ be a $K$-analytic space. Recall that a tropical chart of $X$ is a pair $(f: U \rightarrow T, P)$, where $f: X \rightarrow T$ is a map to a torus $T$ over $K$, usually called a moment map, and $P$ is a compact polyhedral complex of $T_{\text {trop }}$ that contains $f_{\operatorname{trop}}(X)$. Here, $T_{\text {trop }}$ is the tropicalization of $T$, which is a real vector space of finite dimension, and $f_{\text {trop }}: X \rightarrow T \rightarrow T_{\text {trop }}$ is the composite map.

For every open subset $U$ of $X$, let $\mathscr{A}_{\text {pre }}^{p, q}(U)$ be the inductive limit of $\mathscr{A}_{P}^{p, q}(P)$ for all tropical charts $(f: U \rightarrow T, P)$ of $U$. Again by Lemma 2.4, the monodromy maps $\mathrm{N}_{P}$ are compatible with transition maps. Therefore, we obtain a map $\mathrm{N}_{\text {pre }}: \mathscr{A}_{\text {pre }}^{p, q}(U) \rightarrow \mathscr{A}_{\text {pre }}^{p-1, q+1}(U)$ for $p \geqslant 1$. The sheaf of $(p, q)$-forms on $X$ is defined as the sheafification of $U \mapsto \mathscr{A}_{\mathrm{pre}}^{p, q}(U)$ and is denoted by $\mathscr{A}_{X}^{p, q}$.

Definition 3.1. For $p \geqslant 1$, we define the monodromy map for forms on $X$, denoted by

$$
\mathrm{N}_{X}: \mathscr{A}_{X}^{p, q} \rightarrow \mathscr{A}_{X}^{p-1, q+1}
$$

to be the sheafification of the map $U \mapsto\left[\mathrm{N}_{\text {pre }}: \mathscr{A}_{\text {pre }}^{p, q}(U) \rightarrow \mathscr{A}_{\text {pre }}^{p-1, q+1}(U)\right]$ of presheaves. For $0 \leqslant r \leqslant p$, we denote the iterated composition by $\mathrm{N}_{X}^{r}: \mathscr{A}_{X}^{p, q} \rightarrow \mathscr{A}_{X}^{p-r, q+r}$.

Lemma 3.2. (i) The map $\mathrm{N}_{X}^{p}: \mathscr{A}_{X}^{p, q} \rightarrow \mathscr{A}_{X}^{0, p}$ coincides with $p$ ! $\cdot \mathrm{J}$.

(ii) The map $\mathrm{N}_{X}$ commutes with $\mathrm{d}^{\prime \prime}$; that is, $\mathrm{N}_{X} \mathrm{~d}^{\prime \prime}=\mathrm{d}^{\prime \prime} \mathrm{N}_{X}: \mathscr{A}_{X}^{p, q} \rightarrow \mathscr{A}_{X}^{p-1, q+2}$ for $p \geqslant 1$.

(iii) We have $\left(\mathrm{N}_{X} \bullet\right) \wedge-=-\left(\bullet \wedge\left(\mathrm{N}_{X} \bullet\right)\right): \mathscr{A}_{X}^{p, q} \times \mathscr{A}_{X}^{p^{\prime}, q^{\prime}} \rightarrow \mathscr{A}_{X}^{p+p^{\prime}-1, q+q^{\prime}+1}$ for $p, p^{\prime} \geqslant 1$ and $p+p^{\prime} \geqslant \operatorname{dim} X+1$.

(iv) Let $\varphi: X^{\prime} \rightarrow X$ be a map of $K$-analytic spaces. Then

$$
\left(\varphi_{*} \mathrm{~N}_{X^{\prime}}\right) \varphi^{*}=\varphi^{*} \mathrm{~N}_{X}: \mathscr{A}_{X}^{p, q} \rightarrow \varphi_{*} \mathscr{A}_{X^{\prime}}^{p-1, q+1} .
$$

Proof. These are consequences of Lemmas 2.5, 2.2, 2.3, and 2.4, respectively.

\footnotetext{
${ }^{2}$ It is called polytope in [CD12, Section 1.1].
} 


\section{Monodromy MAP FOR TROPICAL DOLBEAUlt COHOMOLOGY}

For a fixed integer $p$, we have the complex

$$
\left(\mathscr{A}_{X}^{p, \bullet}, \mathrm{d}^{\prime \prime}\right): \mathscr{A}_{X}^{p, 0} \stackrel{\mathrm{d}^{\prime \prime}}{\rightarrow} \mathscr{A}_{X}^{p, 1} \stackrel{\mathrm{d}^{\prime \prime}}{\longrightarrow} \cdots .
$$

The monodromy map in Definition 3.1 is in fact a map of complexes

$$
\mathrm{N}_{X}: \mathscr{A}_{X}^{p, \bullet} \rightarrow \mathscr{A}_{X}^{p-1, \bullet}[1]
$$

Definition 3.3 (Dolbeault cohomology, [Liu17, Definition 3.1]). Let $X$ be a $K$-analytic space. We define the Dolbeault cohomology of $X$ to be the sheaf cohomology

$$
\mathrm{H}^{p, q}(X):=\mathrm{H}^{q}\left(X, \operatorname{ker}\left(\mathrm{d}^{\prime \prime}: \mathscr{A}_{X}^{p, 0} \rightarrow \mathscr{A}_{X}^{p, 1}\right)\right) .
$$

Let $\mathscr{O}_{X}$ be the structure sheaf of $X$. For $p \geqslant 0$, let $\mathscr{O}_{X}^{(p)}$ be the sheaf such that for every open subset $U$ of $X$, the set $\mathscr{O}_{X}^{(p)}(U)$ is the $\mathbb{Q}$-vector space spanned by symbols $\left\{f_{1}, \ldots, f_{p}\right\}$ with $f_{i} \in \mathscr{O}_{X}^{*}(U)$. For $p \geqslant 0$, we have a natural map

$$
\tau: \mathscr{O}_{X}^{(p)} \rightarrow \operatorname{ker}\left(\mathrm{d}^{\prime \prime}: \mathscr{A}_{X}^{p, 0} \rightarrow \mathscr{A}_{X}^{p, 1}\right)
$$

of $\mathbb{Q}$-sheaves on $X$. Let $\mathscr{T}_{X}^{p}$ be its image sheaf. We recall the definition of $\tau$. For an open subset $U$ of $X$ and $f_{1}, \ldots, f_{p} \in \mathscr{O}_{X}^{*}(U)$, we have a moment map

$$
f=\left(f_{1}, \ldots, f_{p}\right): U \rightarrow T:=\left(\mathbf{G}_{m}^{\mathrm{an}}\right)^{p} .
$$

Let $\left\{x_{1}, \ldots, x_{p}\right\}$ be the standard coordinates of $T_{\text {trop }}=\mathbb{R}^{p}{ }^{3}$ Then $\tau\left(\left\{f_{1}, \ldots, f_{p}\right\}\right)$ is defined as $\mathrm{d}^{\prime} x_{1} \wedge \cdots \wedge \mathrm{d}^{\prime} x_{p}$, regarded as an element in $\operatorname{ker}\left(\mathrm{d}^{\prime \prime}: \mathscr{A}_{X}^{p, 0}(U) \rightarrow \mathscr{A}_{X}^{p, 1}(U)\right)$.

Proposition 3.4. Let $X$ be a $K$-analytic space. The canonical map

$$
\mathscr{T}_{X}^{p} \otimes_{\mathbb{Q}} \mathbb{R} \rightarrow \operatorname{ker}\left(\mathrm{d}^{\prime \prime}: \mathscr{A}_{X}^{p, 0} \rightarrow \mathscr{A}_{X}^{p, 1}\right)
$$

is an isomorphism. It induces a canonical isomorphism $\mathrm{H}^{q}\left(X, \mathscr{T}_{X}^{p}\right) \otimes_{\mathbb{Q}} \mathbb{R} \simeq \mathrm{H}^{p, q}(X)$.

Proof. This follows from [Liu17, Proposition 3.6 and Corollary 3.7].

Remark 3.5. If $X$ is paracompact, then by [Liu17, Remark 3.2], we have a canonical isomorphism

$$
\mathrm{H}^{p, q}(X) \simeq \frac{\operatorname{ker}\left(\mathrm{d}^{\prime \prime}: \mathscr{A}_{X}^{p, q}(X) \rightarrow \mathscr{A}_{X}^{p, q+1}(X)\right)}{\operatorname{im}\left(\mathrm{d}^{\prime \prime}: \mathscr{A}_{X}^{p, q-1}(X) \rightarrow \mathscr{A}_{X}^{p, q}(X)\right)},
$$

and the monodromy map (3.1) induces a map

$$
\mathrm{N}_{X}: \mathrm{H}^{p, q}(X) \rightarrow \mathrm{H}^{p-1, q+1}(X)
$$

of Dolbeault cohomology when $p \geqslant 1$.

\section{Semistable schemes and cohomological monodromy maps}

In this section, we introduce some constructions for strictly semistable schemes, and review the (cohomological) monodromy maps coming from the weight spectral sequence.

Let $K$ be a discrete non-Archimedean field, that is, a non-Archimedean field with a discrete valuation. Fix a rational prime $\ell$ that is invertible in $\widetilde{K}$. Denote by $K^{\text {ur }} \subset K^{\text {a }}$ the maximal subextension that is unramified over $K$, with residue field $\widetilde{K}^{\text {s }}$, which is a separable closure of $\widetilde{K}$. Let $\mathrm{I}_{K} \subset \operatorname{Gal}\left(K^{\mathrm{a}} / K\right)$ be the inertia subgroup so the quotient group $\operatorname{Gal}\left(K^{\mathrm{a}} / K\right) / \mathrm{I}_{K}$ can be

${ }^{3}$ The map $\mathbf{G}_{m}^{\text {an }} \rightarrow\left(\mathbf{G}_{m}^{\text {an }}\right)_{\text {trop }}=\mathbb{R}$ is given by $-\log ||$. 
identified with $\operatorname{Gal}\left(\widetilde{K}^{\mathrm{s}} / \widetilde{K}\right)$. Denote by $t_{\ell}: \mathrm{I}_{K} \rightarrow \mathbb{Z}_{\ell}(1)$ the ( $\ell$-adic) tame quotient homomorphism; that is, the one sending $\sigma \in \mathrm{I}_{K}$ to $\left(\sigma\left(\varpi^{1 / \ell^{n}}\right) / \varpi^{1 / \ell^{n}}\right)_{n}$ for a uniformizer $\varpi$ of $K$. We fix an element $T \in \mathrm{I}_{K}$ such that $t_{\ell}(T)$ is a topological generator of $\mathbb{Z}_{\ell}(1)$.

For a separated scheme $X$ of finite type over $K$, put $X_{\mathrm{a}}:=X \otimes_{K} K^{\mathrm{a}}$, and let $X^{\text {an }}$ be the associated $K$-analytic space as in [Ber93]. Put $X_{\mathrm{a}}^{\mathrm{an}}:=\left(X \otimes_{K} \widehat{K^{\mathrm{a}}}\right)^{\text {an }}$. For a scheme $\mathcal{X}$ over $K^{\circ}$, put $\mathcal{X}_{\eta}:=\mathcal{X} \otimes_{K^{\circ}} K$. For a scheme $Z$ over $\widetilde{K}$, put $Z_{\mathrm{s}}:=Z \otimes_{\widetilde{K}} \widetilde{K}^{\mathrm{s}}$.

We first recall the following definition (see [Sai03], for example).

Definition 4.1 (Strictly semistable scheme). Let $\mathcal{X}$ be a scheme locally of finite presentation over Spec $K^{\circ}$. We say that $\mathcal{X}$ is strictly semistable if it is Zariski-locally smooth over

$$
\operatorname{Spec} K^{\circ}\left[t_{0}, \ldots, t_{p}\right] /\left(t_{0} \cdots t_{p}-\varpi\right)
$$

for some integer $p \geqslant 0$ (which may vary) and a uniformizer $\varpi$ of $K$.

Let $\mathcal{X}$ be a proper, strictly semistable scheme over $K^{\circ}$. The special fiber $Y:=\mathcal{X} \otimes_{K^{\circ}} \widetilde{K}$ is a normal crossing divisor of $\mathcal{X}$. Suppose that $\left\{Y^{1}, \ldots, Y^{m}\right\}$ is the set of irreducible components of $Y$. For a nonempty subset $I \subset\{1, \ldots, m\}$, put $Y^{I}:=\bigcap_{i \in I} Y^{i}$. For $p \geqslant 0$, put

$$
Y^{(p)}:=\coprod_{I \subset\{1, \ldots, m\},|I|=p+1} Y^{I} .
$$

Then $Y^{(p)}$ is a finite, disjoint union of smooth, proper subschemes of $Y$ of codimension $p$ over $\widetilde{K}$. Denote the image of the canonical morphism $Y^{(p)} \rightarrow Y$ by $Y^{[p]}$.

Notation 4.2. For a subscheme $Z$ of $Y$, put $I(Z):=\left\{i \in\{1, \ldots, m\} \mid Z \subset Y^{i}\right\}$ and $p(Z):=$ $|I(Z)|-1$.

Definition 4.3. Let $y$ be a point of $Y$ with $p=p(y) \geqslant 0$. Suppose $I(y)=\left\{i_{0}<\cdots<i_{p}\right\}$ (Notation 4.2). We define a semistable chart at $y$ to be a pair $\left(\mathcal{V},\left\{g_{0}, \ldots, g_{p}\right\}\right)$, where $\mathcal{V}$ is a connected, affine, open neighborhood of $y$ in $\mathcal{X}$ and $g_{0}, \ldots, g_{p}$ are regular functions on $\mathcal{V}$ such that there is a smooth morphism $\mathcal{V} \rightarrow \operatorname{Spec} K^{\circ}\left[t_{0}, \ldots, t_{p}\right] /\left(t_{0} \cdots t_{p}-\varpi\right)$ under which $g_{j}$ is the pullback of $t_{j}$ and its associated divisor is $\mathcal{V} \cap Y^{i_{j}}$ for $0 \leqslant j \leqslant p$.

Remark 4.4. For every point $y$ of $Y$, one can always find a semistable chart at $y$ by Definition 4.1. Moreover, for every irreducible component $W$ of $Y^{[r]}$ with $r \geqslant 0$, the intersection $\mathcal{V} \cap W$ is either empty or connected since $W$ is smooth and irreducible.

For subsets $J \subset I \subset\{1, \ldots, m\}$ such that $|I|=|J|+1$, let $i_{J I}: \bigcap_{i \in I} Y^{i} \rightarrow \bigcap_{i \in J} Y^{i}$ denote the closed immersion. If $I=\left\{i_{0}<\cdots<i_{p}\right\}$ and $J=I \backslash\left\{i_{j}\right\}$, then we put $\epsilon(J, I)=(-1)^{j}$. We define the pullback map

$$
\delta_{p}^{*}: \mathrm{H}_{\text {ét }}^{q}\left(Y_{\mathrm{s}}^{(p)}, \mathbb{Q}_{\ell}\right) \rightarrow \mathrm{H}_{\text {ét }}^{q}\left(Y_{\mathrm{s}}^{(p+1)}, \mathbb{Q}_{\ell}\right)
$$

to be the alternating sum $\sum_{I \subset J,|I|=|J|-1=p+1} \epsilon(I, J) i_{I J}^{*}$ of the restriction maps and the pushforward map

$$
\delta_{p *}: \mathrm{H}_{\text {ét }}^{q}\left(Y_{\mathrm{s}}^{(p)}, \mathbb{Q}_{\ell}\right) \rightarrow \mathrm{H}_{\text {ét }}^{q+2}\left(Y_{\mathrm{s}}^{(p-1)}, \mathbb{Q}_{\ell}(1)\right)
$$

to be the alternating sum $\sum_{I \supset J,|I|=|J|+1=p+1} \epsilon(J, I) i_{J I *}$ of the Gysin maps. For $p \geqslant 1$, these maps satisfy the formula

$$
\delta_{p-1}^{*} \circ \delta_{p *}+\delta_{p+1 *} \circ \delta_{p}^{*}=0 .
$$




\section{MONODROMY MAP FOR TROPICAL DOLBEAUlT COHOMOLOGY}

Let us recall the following weight spectral sequence $\mathrm{E}_{\mathcal{X}, \bullet}^{\bullet, \bullet}$ attached to $\mathcal{X},{ }^{4}$ originally studied in [RZ82]:

$$
\mathrm{E}_{\mathcal{X}, 1}^{p, q}=\bigoplus_{i \geqslant \max (0,-p)} \mathrm{H}_{\text {ét }}^{q-2 i}\left(Y_{\mathrm{s}}^{(p+2 i)}, \mathbb{Q}_{\ell}(-i)\right) \Rightarrow \mathrm{H}_{\text {ét }}^{p+q}\left(\mathcal{X}_{\eta, \mathrm{a}}, \mathbb{Q}_{\ell}\right) .
$$

Here, we will follow the convention and discussion in [Sai03]. When $\mathcal{X}$ is fixed, we write $\mathrm{E}_{\bullet}^{\bullet \bullet \bullet}$ for $\mathrm{E}_{\mathcal{X}, \bullet}^{\bullet, \bullet}$ for short. By [Sai03, Corollary 2.8(2)], we have a map $\mu: \mathrm{E}_{\bullet}^{\bullet-1, \bullet+1} \rightarrow \mathrm{E}_{\bullet}^{\bullet+1, \bullet-1}$ of spectral sequences (depending on $T$ ). The differential map $d_{1}^{p, q}$ is an appropriate sum of pullback and pushforward maps. The map $\mu_{1}^{p, q}: \mathrm{E}_{1}^{p-1, q+1} \rightarrow \mathrm{E}_{1}^{p+1, q-1}$ is the sum of its restrictions to each direct summand $\mathrm{H}_{\mathrm{ett}}^{q+1-2 i}\left(Y_{\mathrm{s}}^{(2 i+1)}, \mathbb{Q}_{\ell}(-i)\right)$, and such a restriction is the tensor product map by $t_{\ell}(T)$ (respectively, the zero map) if $\mathrm{H}_{\text {ét }}^{q+1-2 i}\left(Y_{\mathrm{s}}^{(2 i+1)}, \mathbb{Q}_{\ell}(-i+1)\right)$ does (respectively, does not) appear in the target. For integers $p, q, r \geqslant 0$, the map $\mu$ induces a map

$$
\mathrm{N}_{\mathcal{X}}^{r}: \mathrm{E}_{2}^{p, q}(r) \rightarrow \mathrm{E}_{2}^{p+2 r, q-2 r},
$$

which depends only on $\mathcal{X}$.

ConjeCture 4.5 (Weight-monodromy conjecture). Let $\mathcal{X}$ be a proper, strictly semistable scheme over $K^{\circ}$. Then for all integers $p, r \geqslant 0$, the map

$$
\mathrm{N}_{\mathcal{X}}^{r}: \mathrm{E}_{2}^{-r, p+r}(r) \rightarrow \mathrm{E}_{2}^{r, p-r}
$$

is an isomorphism.

For $p \geqslant 1$, the map $\mathrm{N}_{\mathcal{X}}^{p}: \mathrm{E}_{2}^{-p, 2 p}(p) \rightarrow \mathrm{E}_{2}^{p, 0}$ is simply the map

$$
\begin{aligned}
\mathrm{E}_{2}^{-p, 2 p}(p) & =\operatorname{ker}\left(\mathrm{H}_{\text {ét }}^{0}\left(Y_{\mathrm{s}}^{(p)}, \mathbb{Q}_{\ell}\right) \stackrel{\left(\delta_{p}^{*}, \delta_{p *}\right)}{\longrightarrow} \mathrm{H}_{\text {ét }}^{0}\left(Y_{\mathrm{s}}^{(p+1)}, \mathbb{Q}_{\ell}\right) \oplus \mathrm{H}_{\text {ét }}^{2}\left(Y_{\mathrm{s}}^{(p-1)}, \mathbb{Q}_{\ell}(1)\right)\right) \\
& \rightarrow \frac{\operatorname{ker}\left(\mathrm{H}_{\text {ét }}^{0}\left(Y_{\mathrm{s}}^{(p)}, \mathbb{Q}_{\ell}\right) \stackrel{\delta_{p}^{*}}{\longrightarrow} \mathrm{H}_{\text {ét }}^{0}\left(Y_{\mathrm{s}}^{(p+1)}, \mathbb{Q}_{\ell}\right)\right)}{\operatorname{im}\left(\mathrm{H}_{\text {ét }}^{0}\left(Y_{\mathrm{s}}^{(p-1)}, \mathbb{Q}_{\ell}\right) \stackrel{\delta_{p-1}^{*}}{\longrightarrow} \mathrm{H}_{\text {ét }}^{0}\left(Y_{\mathrm{s}}^{(p)}, \mathbb{Q}_{\ell}\right)\right)}=\mathrm{E}_{2}^{p, 0}
\end{aligned}
$$

induced by the identity map on $\mathrm{H}_{\text {ét }}^{0}\left(Y_{\mathrm{s}}^{(p)}, \mathbb{Q}_{\ell}\right)$.

Proposition 4.6. (i) The spectral sequence $\mathrm{E}_{\mathcal{X}}$ degenerates from the second page. In particular, $\mathrm{E}_{2}^{p, 0}$ is canonically a subspace of $\mathrm{H}_{\text {ét }}^{p}\left(\mathcal{X}_{\eta, \mathrm{a}}, \mathbb{Q}_{\ell}\right)$.

(ii) If $K$ has equal characteristic, then Conjecture 4.5 holds.

(iii) If $\widetilde{K}$ is a purely inseparable extension of a finitely generated extension of a prime field, then $\mathrm{N}_{\mathcal{X}}: \mathrm{E}_{2}^{-1,2}(1) \rightarrow \mathrm{E}_{2}^{1,0}$ is an isomorphism.

Proof. See [Ito05, Theorem 1.1] for parts (i) and (ii). Part (iii) follows from [Ito05, Proposition 2.5 and Remark 2.4].

Let $X$ be a separated scheme of finite type over $K$. From [Ber00, Section 1], we have the map

$$
\kappa_{X}^{p}: \mathrm{H}^{p}\left(X^{\mathrm{an}}, \mathbb{Q}_{\ell}\right) \rightarrow \mathrm{H}_{\text {ét }}^{p}\left(X_{\mathrm{a}}, \mathbb{Q}_{\ell}\right) .
$$

It is defined as the composition of the restriction map $\mathrm{H}^{p}\left(X^{\mathrm{an}}, \mathbb{Q}_{\ell}\right) \rightarrow \mathrm{H}_{\text {ét }}^{p}\left(X_{\mathrm{a}}^{\mathrm{an}}, \mathbb{Q}_{\ell}\right)$ and the inverse of the comparison isomorphism $\mathrm{H}_{\text {ét }}^{p}\left(X_{\mathrm{a}}, \mathbb{Q}_{\ell}\right) \stackrel{\sim}{\rightarrow} \mathrm{H}_{\text {ét }}^{p}\left(X_{\mathrm{a}}^{\mathrm{an}}, \mathbb{Q}_{\ell}\right)$.

${ }^{4}$ It also depends on the ordering of the set of irreducible components of $Y$. 
Lemma 4.7. The map $\kappa_{\mathcal{X}_{\eta}}^{p}$ in (4.5) is injective with image contained in $\mathrm{E}_{2}^{p, 0}$. Moreover, if every irreducible component of $Y^{(r)}(r \geqslant 0)$ is geometrically irreducible, then $\kappa_{\mathcal{X}_{\eta}}^{p}$ induces an isomorphism $\mathrm{H}^{p}\left(\mathcal{X}_{\eta}^{\text {an }}, \mathbb{Q}_{\ell}\right) \stackrel{\sim}{\rightarrow} \mathrm{E}_{2}^{p, 0}$.

Proof. From [Ber00, Section 4], we have the diagram

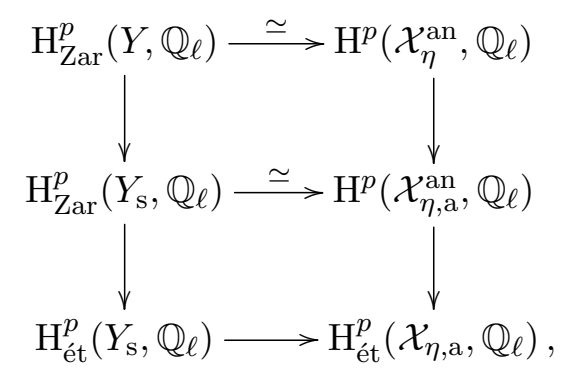

in which the composition of the two right vertical maps is just $\kappa_{\mathcal{X}_{\eta}}^{p}$. By [Ber00, Lemma 4.1], the upper and middle horizontal maps are both isomorphisms. By the discussion after [Ber00, Theorem 1.1], the map $\mathrm{H}^{p}\left(\mathcal{X}_{\eta}^{\text {an }}, \mathbb{Q}_{\ell}\right) \rightarrow \mathrm{H}^{p}\left(\mathcal{X}_{\eta, \mathrm{a}}^{\text {an }}, \mathbb{Q}_{\ell}\right)$ and therefore the map $\mathrm{H}_{\text {Zar }}^{p}\left(Y, \mathbb{Q}_{\ell}\right) \rightarrow \mathrm{H}_{\text {Zar }}^{p}\left(Y_{\mathrm{s}}, \mathbb{Q}_{\ell}\right)$ are injective. Now we compute the composite map

$$
\mathrm{H}_{\text {Zar }}^{p}\left(Y_{\mathrm{s}}, \mathbb{Q}_{\ell}\right) \rightarrow \mathrm{H}_{\text {ét }}^{p}\left(Y_{\mathrm{s}}, \mathbb{Q}_{\ell}\right) \rightarrow \mathrm{H}_{\text {ét }}^{p}\left(\mathcal{X}_{\eta, \mathrm{a}}, \mathbb{Q}_{\ell}\right) .
$$

To compute the term $\mathrm{H}_{\mathrm{Zar}}^{p}\left(Y_{\mathrm{s}}, \mathbb{Q}_{\ell}\right)$, we apply the topological proper descent theorem [SGA4-2, Exposé vbis, Corollaire 4.1.6] to the covering $\coprod_{i=1}^{m} Y_{\mathrm{s}}^{i} \rightarrow Y_{\mathrm{s}}$. Note that for every smooth scheme $Z$ over $\widetilde{K}^{\text {s }}$, the cohomology $\mathrm{H}_{\mathrm{Zar}}^{i}\left(Z, \mathbb{Q}_{\ell}\right)$ vanishes for $i>0$ as constant sheaves are flasque over $Z_{\mathrm{Zar}}$. By the descent spectral sequence, we have a canonical isomorphism

$$
\mathrm{H}_{\mathrm{Zar}}^{p}\left(Y_{\mathrm{s}}, \mathbb{Q}_{\ell}\right) \simeq \frac{\operatorname{ker}\left(\mathrm{H}_{\mathrm{Zar}}^{0}\left(Y_{\mathrm{s}}^{(p)}, \mathbb{Q}_{\ell}\right) \stackrel{\delta_{p}^{*}}{\longrightarrow} \mathrm{H}_{\mathrm{Zar}}^{0}\left(Y_{\mathrm{s}}^{(p+1)}, \mathbb{Q}_{\ell}\right)\right)}{\operatorname{im}\left(\mathrm{H}_{\mathrm{Zar}}^{0}\left(Y_{\mathrm{s}}^{(p-1)}, \mathbb{Q}_{\ell}\right) \stackrel{\delta_{p-1}^{*}}{\longrightarrow} \mathrm{H}_{\mathrm{Zar}}^{0}\left(Y_{\mathrm{s}}^{(p)}, \mathbb{Q}_{\ell}\right)\right)}
$$

Thus, the map (4.6) is an isomorphism onto its image, which is $\mathrm{E}_{2}^{p, 0}$. Therefore, the map $\kappa_{\mathcal{X}_{\eta}}^{p}$ from (4.5) is injective with image contained in $\mathrm{E}_{2}^{p, 0}$.

Moreover, if every irreducible component of $Y^{(r)}$ is geometrically irreducible, then the restriction map $\mathrm{H}_{\mathrm{Zar}}^{0}\left(Y^{(p)}, \mathbb{Q}_{\ell}\right) \rightarrow \mathrm{H}_{\mathrm{Zar}}^{0}\left(Y_{\mathrm{s}}^{(p)}, \mathbb{Q}_{\ell}\right)$ is an isomorphism for every $p \geqslant 0$. Thus, again by topological proper descent, the map $\mathrm{H}_{\mathrm{Zar}}^{p}\left(Y, \mathbb{Q}_{\ell}\right) \rightarrow \mathrm{H}_{\mathrm{Zar}}^{p}\left(Y_{\mathrm{S}}, \mathbb{Q}_{\ell}\right)$ is an isomorphism. The lemma follows.

LEMma 4.8. Suppose that one of following conditions holds:

(i) The field $K$ is local non-Archimedean.

(ii) There is a finite extension $K^{\prime}$ of $K$ such that $X \otimes_{K} K^{\prime}$ is the generic fiber of a proper strictly semistable scheme over $K^{\prime 0}$.

Then the map $\kappa_{X}^{p}$ is injective for all $p \geqslant 0$.

Proof. Case (i) follows from [Ber00, Corollary 1.2]. Case (ii) follows from Lemma 4.7 and the fact that the map $\mathrm{H}^{p}\left(X^{\text {an }}, \mathbb{Q}_{\ell}\right) \rightarrow \mathrm{H}^{p}\left(\left(X \otimes_{K} K^{\prime}\right)^{\text {an }}, \mathbb{Q}_{\ell}\right)$ is injective. 


\section{MONODROMY MAP FOR TROPICAL DOLBEAUlt COHOMOLOGY}

\section{Monodromy maps for tropical Dolbeault cohomology}

In this section, we propose the conjecture on the isomorphism of tropical Dolbeault cohomology groups under monodromy maps. Then, we prove our main results.

Let $K$ be a non-Archimedean field. Let $X$ be a separated scheme of finite type over $K$. Note that $X^{\text {an }}$ is paracompact.

Definition 5.1 (Tropical Dolbeault cohomology, [Liu17]). We define the tropical Dolbeault cohomology of $X$ to be

$$
\mathrm{H}_{\text {trop }}^{p, q}(X):=\mathrm{H}^{q}\left(X^{\text {an }}, \mathscr{T}_{X^{\text {an }}}^{p}\right) .
$$

Then $\mathrm{H}_{\text {trop }}^{p, q}(X)_{\mathbb{R}}$ is canonically isomorphic to $\mathrm{H}^{p, q}\left(X^{\text {an }}\right)$ by Proposition 3.4. We define the corresponding tropical Hodge number of $X$ to be

$$
\mathrm{h}_{\text {trop }}^{p, q}(X):=\operatorname{dim}_{\mathbb{Q}} \mathrm{H}_{\text {trop }}^{p, q}(X) .
$$

We have the monodromy map

$$
\mathrm{N}_{X}:=\mathrm{N}_{X} \text { an }: \mathrm{H}_{\text {trop }}^{p, q}(X)_{\mathbb{R}} \rightarrow \mathrm{H}_{\text {trop }}^{p-1, q+1}(X)_{\mathbb{R}}
$$

for $p \geqslant 1$ by Remark 3.5.

Remark 5.2. Since $\mathscr{T}_{X^{\text {an }}}^{0}$ is the constant sheaf $\mathbb{Q}$, we have $\mathrm{H}_{\text {trop }}^{0, q}(X)=\mathrm{H}^{q}\left(X^{\text {an }}, \mathbb{Q}\right)$.

CONJECTURE 5.3. Suppose that $K$ is an algebraically closed, non-Archimedean field such that $\widetilde{K}$ is algebraic over a finite field. Let $X$ be a proper, smooth scheme over $K$. Then for $p \geqslant q \geqslant 0$, the (iterated) monodromy map

$$
\mathrm{N}_{X}^{p-q}: \mathrm{H}_{\text {trop }}^{p, q}(X)_{\mathbb{R}} \rightarrow \mathrm{H}_{\text {trop }}^{q, p}(X)_{\mathbb{R}}
$$

is an isomorphism.

Remark 5.4. Conjecture 5.3 does not hold for arbitrary algebraically closed non-Archimedean fields. In fact, let $X_{0}$ be the generic fiber of the scheme $\mathfrak{X}$ in [BGS95, Section (6.1)], which is a geometrically connected, projective, smooth curve over $K_{0}:=\mathbb{C}((t))$. Now take $K=\widehat{K_{0}^{\mathrm{a}}}$ and $X=X_{0} \otimes_{K_{0}} K$. One can show that $\mathrm{h}_{\text {trop }}^{1,0}(X)=0$ but $\mathrm{h}_{\text {trop }}^{0,1}(X)=2$. This also implies that the canonical pairing

$$
\mathrm{H}_{\text {trop }}^{1,0}(X)_{\mathbb{R}} \times \mathrm{H}_{\text {trop }}^{0,1}(X)_{\mathbb{R}} \rightarrow \mathrm{H}_{\text {trop }}^{1,1}(X)_{\mathbb{R}} \stackrel{\operatorname{Tr}}{\longrightarrow} \mathbb{R}
$$

(where $\mathrm{Tr}$ is the integration map) is not a perfect pairing; in other words, the Poincaré duality fails.

Definition 5.5. Let $X$ be a proper, smooth scheme over a discrete non-Archimedean field $K$. A strict semistable alteration of $X$ is a proper, strictly semistable scheme $\mathcal{X}$ over $K_{\mathcal{X}}^{\circ}$ for some finite extension $K_{\mathcal{X}} / K$ contained in $K^{\mathrm{a}}$, together with a proper, generically étale morphism $\phi: \mathcal{X}_{\eta} \rightarrow X$ over $K$, such that every irreducible component of $Y^{(r)}$ for $r \geqslant 0$ is geometrically irreducible.

Theorem 5.6. Let $X$ be a proper, smooth scheme over a discrete non-Archimedean field $K$ and $p \geqslant 0$ an integer. Suppose that the map $\kappa_{X}^{p}$ from (4.5) is injective.

(i) If $\log |x| \in \mathbb{Q}$ for every $x \in K^{\times}$, then the monodromy map

$$
\mathrm{N}_{X}^{p}: \mathrm{H}_{\text {trop }}^{p, 0}(X)_{\mathbb{R}} \rightarrow \mathrm{H}_{\text {trop }}^{0, p}(X)_{\mathbb{R}}
$$

preserves the rational structure; that is, it sends $\mathrm{H}_{\text {trop }}^{p, 0}(X)$ into $\mathrm{H}_{\text {trop }}^{0, p}(X)$. 
(ii) Suppose that for every strict semistable alteration $\mathcal{X}$ of $X$ (Definition 5.5), the map

$$
\mathrm{N}_{\mathcal{X}}^{p}: \mathrm{E}_{2}^{-p, 2 p}(p) \rightarrow \mathrm{E}_{2}^{p, 0}
$$

from (4.3) is injective. Then $\mathrm{N}_{X}^{p}: \mathrm{H}_{\text {trop }}^{p, 0}(X)_{\mathbb{R}} \rightarrow \mathrm{H}_{\text {trop }}^{0, p}(X)_{\mathbb{R}}$ is injective.

We need some preparation before the proof. The case $p=0$ is trivial. So we assume $p \geqslant 1$. The following notation will be used later.

Notation 5.7. Let $h: T^{\prime} \rightarrow T$ be a homomorphism of $K$-analytic torus. Then we denote by $h^{b}: T_{\text {trop }}^{\prime} \rightarrow T_{\text {trop }}$ the induced linear map under tropicalization.

For a proper, strictly semistable scheme $\mathcal{X}$ over $K_{\mathcal{X}}^{\circ}$, recall that we have the reduction map

$$
\pi_{\mathcal{X}}: \mathcal{X}_{\eta}^{\mathrm{an}} \rightarrow Y
$$

where $Y$ is the special fiber of $\mathcal{X}$ as before.

Let $\omega$ be an element in $\mathrm{H}_{\text {trop }}^{p, 0}(X)=\mathrm{H}^{0}\left(X^{\text {an }}, \mathscr{T}_{X^{\text {an }}}^{p}\right)$. We say that a strict semistable alteration $\mathcal{X}$ of $X$ (with a morphism $\phi: \mathcal{X}_{\eta} \rightarrow X$ ) presents $\omega$ if for every irreducible component $Y^{i}$ of $Y$, there exist

- an open subset $U$ of $\mathcal{X}_{\eta}^{\text {an }}$ containing $\pi_{\mathcal{X}}^{-1} Y^{i}$,

- $c_{l} \in \mathbb{Q}$ for $1 \leqslant l \leqslant M$ with some integer $M=M_{U} \geqslant 1$,

$-f_{l k} \in \mathscr{O}_{\mathcal{X}_{\eta}^{\text {an }}}^{*}(U)$ for $1 \leqslant l \leqslant M$ and $1 \leqslant k \leqslant p$ satisfying $\left|f_{l k}\right|=1$ on $\pi_{\mathcal{X}}^{-1}\left(Y^{i} \backslash Y^{[1]}\right)$

such that

$$
\left.\phi^{\mathrm{an} *} \omega\right|_{U}=\tau\left(\sum_{l=1}^{M} c_{l}\left\{f_{l 1}, \ldots, f_{l p}\right\}\right),
$$

where $\tau$ is the map (3.2). We call such data $\left(U,\left\{c_{l}\right\},\left\{f_{l k}\right\}\right)$ a presentation of $\omega$ on $Y^{i}$.

Now let $Z$ be an irreducible component of $Y^{I}$ for some $I=\left\{i_{0}<\cdots<i_{p}\right\}$ with $p \geqslant 1$. Choose a presentation $\left(U,\left\{c_{l}\right\},\left\{f_{l k}\right\}\right)$ of $\omega$ on $Y^{i_{0}}$. For $j=1, \ldots, p$, let $a_{l k j} \in \mathbb{Z}$ be the order of zero (or the negative order of pole) of $f_{l k}$ along the connected component of $Y^{i_{0}} \cap Y^{i_{j}}$ containing $Z$.

Lemma 5.8. Let the notation be as above. The rational number $\sum_{l=1}^{M} c_{l} \operatorname{det}\left(a_{l k j}\right)_{k, j=1}^{p} \operatorname{depends}$ only on $\phi^{\text {an* } \omega}$ and $Z$.

Proof. Let $\eta_{Z}$ be the generic point of $Z$. Then $I\left(\eta_{Z}\right)=\left\{i_{0}<\cdots<i_{p}\right\}$ (Notation 4.2). We choose a semistable chart $\left(\mathcal{V},\left\{g_{0}, \ldots, g_{p}\right\}\right)$ of $\eta_{Z}$ (Definition 4.3). Put $V:=\mathcal{V} \cap Y^{i_{0}}$. Since $U$ contains $\pi_{\mathcal{X}}^{-1} Y^{i_{0}}$, since $\mathcal{V}_{\eta}^{\text {an }}$ contains $\pi_{\mathcal{X}}^{-1}(\mathcal{V} \cap Y)$, and since both $U$ and $\mathcal{V}_{\eta}^{\text {an }}$ are open in $\mathcal{X}_{\eta}^{\text {an }}$, we know that $U \cap \mathcal{V}_{\eta}^{\text {an }}$ is an open neighborhood of $\pi_{\mathcal{X}}^{-1} V$. Now we replace $U$ by $U \cap \mathcal{V}_{\eta}^{\text {an }}$ and regard $g_{1}, \ldots, g_{p}$ as elements in $\mathscr{O}_{\mathcal{X}}^{*}$ an $(U)$. Then for $1 \leqslant j \leqslant p$, we have $\left|g_{j}\right|=1$ on $\pi_{\mathcal{X}}^{-1}\left(V \backslash Y^{[1]}\right)$, and the divisor associated with the reduction $\widetilde{g_{j}}$ on $V$ is $V \cap Y^{i_{j}}$.

Note that the divisor associated with $\left.\widetilde{f_{l k}}\right|_{V}$ is supported on $V \cap\left(\bigcup_{j=1}^{p} Y^{i_{j}}\right)$. Put

$$
f_{l k}^{\prime}=f_{l k} \cdot \prod_{j=1}^{p} g_{j}^{-a_{l k j}}
$$

Then the reduction $\widetilde{f_{l k}^{\prime}}$ is invertible on $V$. In other words, $\left|f_{l k}^{\prime}\right|=1$ on $\pi_{\mathcal{X}}^{-1} V$. Thus, we have 


\section{MONODROMY MAP FOR TROPICAL DOLBEAUlt COHOMOLOGY}

$\tau\left(\left\{f_{l k}^{\prime}\right\}\right)=0 \in \mathbb{R}$ on $\pi_{\mathcal{X}}^{-1} V$. An elementary calculation shows that

$$
\left.\phi^{\mathrm{an} *} \omega\right|_{\pi_{\mathcal{X}}^{-1} V}=\tau\left(\sum_{l=1}^{M} c_{l}\left\{f_{l 1}, \ldots, f_{l p}\right\}\right)=\left(\sum_{l=1}^{M} c_{l} \operatorname{det}\left(a_{l k j}\right)_{k, j=1}^{p}\right) \tau\left(\left\{g_{1}, \ldots, g_{p}\right\}\right) .
$$

It is clear that $\tau\left(\left\{g_{1}, \ldots, g_{p}\right\}\right)$ is nonzero as $V$ contains $\eta_{Z}$. Since the choice of the semistable chart is independent of the presentation $\omega$ on $Y^{i_{0}}$, the lemma then follows.

We denote the rational number in Lemma 5.8 by $\operatorname{ord}_{\omega}(Z)$. The assignment $Z \mapsto \operatorname{ord}_{\omega}(Z)$ gives rise to an element $\operatorname{ord}_{\omega} \in \mathrm{H}_{\text {ét }}^{0}\left(Y_{\mathrm{s}}^{(p)}, \mathbb{Q}_{\ell}\right)$. Denote by $\mathrm{H}_{\text {trop }}^{p, 0}(X)_{\mathcal{X}}$ the subset of $\mathrm{H}_{\text {trop }}^{p, 0}(X)$ consisting of the $\omega$ which $\mathcal{X}$ presents. Then it is easy to see that $\mathrm{H}_{\text {trop }}^{p, 0}(X)_{\mathcal{X}}$ is a $\mathbb{Q}$-subspace of $\mathrm{H}_{\text {trop }}^{p, 0}(X)$.

Lemma 5.9. Let ord: $\mathrm{H}_{\text {trop }}^{p, 0}(X)_{\mathcal{X}} \rightarrow \mathrm{H}_{\text {ét }}^{0}\left(Y_{\mathrm{s}}^{(p)}, \mathbb{Q}_{\ell}\right)$ be the map sending $\omega$ to $\operatorname{ord}_{\omega}$.

(i) The map ord is injective.

(ii) The image of ord is contained in the subspace $\mathrm{E}_{2}^{-p, 2 p}(p) \subset \mathrm{H}_{\text {ét }}^{0}\left(Y_{\mathrm{s}}^{(p)}, \mathbb{Q}_{\ell}\right)$ defined in (4.4).

Proof. For statement (i), take an element $\omega \in \mathrm{H}_{\text {trop }}^{p, 0}(X)_{\mathcal{X}}$. Let $x \in \mathcal{X}_{\eta}^{\text {an }}$ be a point, and put $y:=\pi_{\mathcal{X}}(x) \in Y$. Suppose $I(y)=\left\{i_{0}<\cdots<i_{r}\right\}$ (Notation 4.2) for some $r \geqslant 0$, and choose a semistable chart $\left(\mathcal{V},\left\{g_{0}, \ldots, g_{r}\right\}\right)$ of $y$ (Definition 4.3). Put $V:=\mathcal{V} \cap Y^{i_{0}}$.

Choose a presentation $\left(U,\left\{c_{l}\right\},\left\{f_{l k}\right\}\right)$ of $\omega$ on $Y^{i_{0}}$. As in the proof of Lemma 5.8, we replace $U$ with $U \cap \mathcal{V}_{\eta}^{\text {an }}$ and view $g_{1}, \ldots, g_{r}$ as being in $\mathscr{O}_{\mathcal{X}_{\eta}^{\text {an }}}^{*}(U)$. Then there exist unique integers $a_{l k j}$ such that if we put $f_{l k}^{\prime}:=\prod_{j=1}^{r} g_{j}^{a_{l k j}}$, then $\left|f_{l k}^{\prime}\right|=\left|f_{l k}\right|$ on $\pi_{\mathcal{X}}^{-1} V$. Let $f$ (respectively, $f^{\prime}$ ) be the moment map $\pi_{\mathcal{X}}^{-1} V \rightarrow\left(\mathbf{G}_{m}^{\text {an }}\right)^{M p}$ induced by $\left\{f_{l k}\right\}$ (respectively, $\left.\left\{f_{l k}^{\prime}\right\}\right)$. Then $f_{\text {trop }}=f_{\text {trop }}^{\prime}$. In particular,

$$
\left.\phi^{\mathrm{an} *} \omega\right|_{\pi_{\mathcal{X}}^{-1} V}=\tau\left(\sum_{l=1}^{M} c_{l}\left\{f_{l 1}^{\prime}, \ldots, f_{l p}^{\prime}\right\}\right) .
$$

Let $g: \pi_{\mathcal{X}}^{-1} V \rightarrow\left(\mathbf{G}_{m}^{\text {an }}\right)^{r}$ be the moment map induced by $\left\{g_{1}, \ldots, g_{r}\right\}$. Then there exists a unique homomorphism $h:\left(\mathbf{G}_{m}^{\text {an }}\right)^{r} \rightarrow\left(\mathbf{G}_{m}^{\text {an }}\right)^{M p}$, determined by integers $\left\{a_{l k j}\right\}$ such that $f_{\text {trop }}=(h \circ$

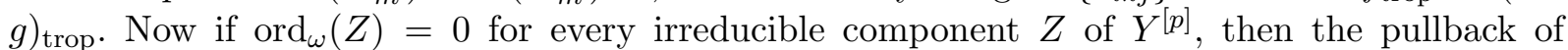
$\tau\left(\sum_{l=1}^{M} c_{l}\left\{f_{l 1}^{\prime}, \ldots, f_{l p}^{\prime}\right\}\right)$ under $h^{b}$ (Notation 5.7) is zero. In other words, we have $\phi^{\text {an* }\left.\omega\right|_{\pi_{\mathcal{X}}^{-1} V}=0}$ by the equality (5.1). Thus, the map ord: $\mathrm{H}_{\text {trop }}^{p, 0}(X)_{\mathcal{X}} \rightarrow \mathrm{H}_{\text {ét }}^{0}\left(Y_{\mathrm{s}}^{(p)}, \mathbb{Q}_{\ell}\right)$ is injective since $\pi_{\mathcal{X}}^{-1} V$ contains $x$ and $x$ is arbitrary; statement (i) follows.

For statement (ii), we need to show that $\delta_{p *} \operatorname{ord}_{\omega}=\delta_{p}^{*} \operatorname{ord}_{\omega}=0$. Suppose that $Z$ is an irreducible component of $Y^{I}$ for some $I=\left\{i_{0}<\cdots<i_{p}\right\}$. For every permutation $\sigma$ of the set $\{0, \ldots, p\}$, we may define in the same way a rational number $\operatorname{ord}_{\omega}^{\sigma}(Z)$ by replacing $i_{j}$ by $i_{\sigma(j)}$. If $\sigma(0)=0$, then we let $\epsilon(\sigma) \in\{ \pm 1\}$ be the sign of the permutation $\left.\sigma\right|_{\{1, \ldots, p\}}$. If $\sigma(0) \neq 0$, then we let $\epsilon(\sigma) \in\{ \pm 1\}$ be the negative of the sign of the permutation from $\{1, \ldots, 0, \ldots, p\}$ (where $\sigma(0)$ is replaced by 0$)$ to $\{\sigma(1), \ldots, \sigma(p)\}$. Then a calculation similar to that in the proof of Lemma 5.8 implies

$$
\operatorname{ord}_{\omega}^{\sigma}(Z)=\epsilon(\sigma) \cdot \operatorname{ord}_{\omega}(Z) .
$$

We start from $\delta_{p *} \operatorname{ord}_{\omega}$. Fix an irreducible component $W$ of $Y^{J}$ for some $J=\left\{i_{0}<\cdots<i_{p-1}\right\}$. For $1 \leqslant j \leqslant p-1$, let $W_{j}$ be the unique irreducible component of $Y^{i_{0}} \cap Y^{i_{j}}$ that contains $W$. Choose a presentation $\left(U,\left\{c_{l}\right\},\left\{f_{l k}\right\}\right)$ of $\omega$ on $Y^{i_{0}}$. By linear algebra, it is easy to see that we may choose $f_{l k}$ such that $\operatorname{ord}_{W_{j}}\left(\widetilde{f_{l k}}\right)=0$ if $j<k$. In particular, the restriction of $\widetilde{f_{l p}}$ on $W$ is a nonzero 
rational function, which we denote by $f_{l p}^{W}$. Put $b_{l, j}=\operatorname{ord}_{W_{j}}\left(\widetilde{f_{l j}}\right)$ for $1 \leqslant j \leqslant p-1$. Let $Z$ be an irreducible component of $W \cap Y[p]$. Then there is a unique element $i_{p} \in\{1, \ldots, m\} \backslash\left\{i_{0}, \ldots, i_{p-1}\right\}$ such that $Z \subset W \cap Y^{i_{p}}$. Let $0 \leqslant \epsilon_{Z} \leqslant p$ be the integer such that there are exactly $\epsilon_{Z}$ elements in $\left\{i_{0}, \ldots, i_{p-1}\right\}$ that are greater than $i_{p}$. Then by (5.2), we have

$$
\operatorname{ord}_{\omega}(Z)=(-1)^{\epsilon_{Z}} \sum_{l=1}^{M} c_{l} b_{l, 1} \cdots b_{l, p-1} \operatorname{ord}_{Z}\left(f_{l p}^{W}\right) .
$$

Therefore, we have the equality

$$
(-1)^{p-\epsilon_{Z}} \sum_{Z \subset W} \operatorname{ord}_{\omega}(Z) Z=(-1)^{p} \sum_{l=1}^{M} c_{l} b_{l, 1} \cdots b_{l, p-1} \operatorname{div}\left(f_{l p}^{W}\right)
$$

of divisors on $W$. By the definition (4.2) of $\delta_{p *}$, the value of $\delta_{p *} \operatorname{ord}_{\omega}$ on $W$, which is an element in $\mathrm{H}_{\text {ét }}^{2}\left(W_{\mathrm{s}}, \mathbb{Q}_{\ell}(1)\right)$, is equal to the cycle class of the left-hand side of (5.3). Since $\operatorname{div}\left(f_{l p}^{W}\right)=0$ in $\mathrm{H}_{\text {ét }}^{2}\left(W_{\mathrm{s}}, \mathbb{Q}_{\ell}(1)\right)$, we have $\delta_{p *} \operatorname{ord}_{\omega}=0$ by $(5.3)$.

Now we consider $\delta_{p}^{*}$ ord $\omega$. Let $W$ be an irreducible component of $Y^{J}$ for some $J=\left\{i_{0}<\cdots<\right.$ $\left.i_{p+1}\right\}$. For $0 \leqslant j \leqslant p+1$, let $Z_{j}$ be the unique irreducible component of $Y^{J \backslash\left\{i_{j}\right\}}$ containing $W$. Let $\left(\mathcal{V},\left\{g_{0}, \ldots, g_{p+1}\right\}\right)$ be a semistable chart of the generic point of $W$ (Definition 4.3). Put $V=\mathcal{V} \cap Y$ and $V_{j}=V \backslash Y^{i_{j}}$. From the proof of part (i), we know that

$$
\left.\phi^{\mathrm{an} *} \omega\right|_{\pi_{\mathcal{X}}^{-1} V}=\sum_{\alpha} c_{\alpha} \tau\left(\left\{g_{\alpha(1)}, \ldots, g_{\alpha(p)}\right\}\right)
$$

for some $c_{\alpha} \in \mathbb{Q}$, where the sum is taken over all strictly increasing maps $\alpha:\{1, \ldots, p\} \rightarrow$ $\{0, \ldots, p+1\}$. We take such a map $\alpha$. Now let $\alpha_{0}<\alpha_{1}$ be the two integers in $\{0, \ldots, p+1\}$ not in the image of $\alpha$. We have three cases:

- If $j \in \operatorname{im}(\alpha)$, then $\left.\tau\left(\left\{g_{\alpha(1)}, \ldots, g_{\alpha(p)}\right\}\right)\right|_{\pi_{\mathcal{X}}^{-1} V_{j}}=0$.

- If $j=\alpha_{0}$, then $\left.\tau\left(\left\{g_{\alpha(1)}, \ldots, g_{\alpha(p)}\right\}\right)\right|_{\pi_{\mathcal{X}}^{-1} V_{j}}=\left.(-1)^{\alpha_{1}-1} \tau\left(\left\{g_{\alpha^{\prime}(1)}, \ldots, g_{\alpha^{\prime}(p)}\right\}\right)\right|_{\pi_{\mathcal{X}}^{-1} V_{j}}$, where $\alpha^{\prime}$ is the unique map whose image does not include $\alpha_{0}$ and $\min \left(\{0, \ldots, p+1\} \backslash\left\{\alpha_{0}\right\}\right)$.

- If $j=\alpha_{1}$, then $\left.\tau\left(\left\{g_{\alpha(1)}, \ldots, g_{\alpha(p)}\right\}\right)\right|_{\pi_{\mathcal{X}}^{-1} V_{j}}=\left.(-1)^{\alpha_{0}} \tau\left(\left\{g_{\alpha^{\prime}(1)}, \ldots, g_{\alpha^{\prime}(p)}\right\}\right)\right|_{\pi_{\mathcal{X}}^{-1} V_{j}}$, where $\alpha^{\prime}$ is the unique map whose image does not include $\left\{0, \alpha_{1}\right\}$.

Now we may choose an open affine subscheme $\mathcal{V}_{j}$ of $\mathcal{V}$ with $\mathcal{V}_{j} \cap Y=V_{j}$ such that $\left(\mathcal{V}_{j},\left\{g_{0}, \ldots\right.\right.$, $\left.\left.\widehat{g_{j}}, \ldots, g_{p+1}\right\}\right)$ is a semistable chart of the generic point of $Z_{j}$. Then the above three cases imply that

$$
\left.\phi^{\mathrm{an} *} \omega\right|_{\pi_{\mathcal{X}}^{-1} V_{j}}=\left(\sum_{\alpha, \alpha_{0}=j} c_{\alpha}(-1)^{\alpha_{1}-1}+\sum_{\alpha, \alpha_{1}=j} c_{\alpha}(-1)^{\alpha_{0}}\right) \tau\left(\left\{g_{1}, \ldots, \widehat{g_{j}}, \ldots, g_{p+1}\right\}\right) .
$$

Comparing with the formula (5.1), we have

$$
\operatorname{ord}_{\omega}\left(Z_{j}\right)=\sum_{\alpha, \alpha_{0}=j} c_{\alpha}(-1)^{\alpha_{1}-1}+\sum_{\alpha, \alpha_{1}=j} c_{\alpha}(-1)^{\alpha_{0}} .
$$

However, by (4.1), we have

$$
\left.\delta_{p}^{*} \omega\right|_{W}=\sum_{j=0}^{p+1}(-1)^{j} \operatorname{ord}_{\omega}\left(Z_{j}\right)=\sum_{\alpha} c_{\alpha}(-1)^{\alpha_{0}+\alpha_{1}-1}+c_{\alpha}(-1)^{\alpha_{0}+\alpha_{1}}=0 .
$$

Thus, part (ii) follows. 


\section{MONODROMY MAP FOR TROPICAL DOLBEAUlt COHOMOLOGY}

The map ord in Lemma 5.9 is inspired by and closely related to the construction in [Liu17, Section 4]. The following lemma is the key step to the proof of Theorem 5.6. It also justifies the terminology monodromy map for $\mathrm{N}_{X}$ in Definitions 3.1 and 5.1.

Lemma 5.10. Let $\mathcal{X}$ be a strict semistable alteration of $X$, with $\phi: \mathcal{X}_{\eta} \rightarrow X$. Suppose that we have $\log |\varpi|=-1$ for a uniformizer $\varpi$ of $K$. Then the image of the restriction of $\mathrm{N}_{X}^{p}: \mathrm{H}_{\text {trop }}^{p, 0}(X)_{\mathbb{R}} \rightarrow$ $\mathrm{H}_{\text {trop }}^{0, p}(X)_{\mathbb{R}}$ to $\mathrm{H}_{\text {trop }}^{p, 0}(X)_{\mathcal{X}}$ is contained in $\mathrm{H}_{\text {trop }}^{0, p}(X)=\mathrm{H}^{p}\left(X^{\text {an }}, \mathbb{Q}\right)$ (Remark 5.2). Moreover, the diagram

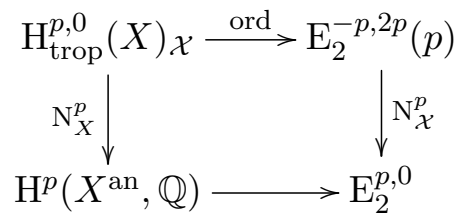

commutes. Here, the bottom map is the composition of

- the multiplication map $(-1)^{p(p+1) / 2}: \mathrm{H}^{p}\left(X^{\mathrm{an}}, \mathbb{Q}\right) \rightarrow \mathrm{H}^{p}\left(X^{\mathrm{an}}, \mathbb{Q}_{\ell}\right)$,

- the map $\kappa_{X}^{p}: \mathrm{H}^{p}\left(X^{\mathrm{an}}, \mathbb{Q}_{\ell}\right) \rightarrow \mathrm{H}_{\text {ét }}^{p}\left(X_{\mathrm{a}}, \mathbb{Q}_{\ell}\right)$ defined in (4.5) (which is assumed to be injective), and

- the pullback map $\phi^{*}: \mathrm{H}_{\text {ét }}^{p}\left(X_{\mathrm{a}}, \mathbb{Q}_{\ell}\right) \rightarrow \mathrm{H}_{\text {ét }}^{p}\left(\mathcal{X}_{\eta, \mathrm{a}}, \mathbb{Q}_{\ell}\right)$.

The image of the bottom map is contained in $\mathrm{E}_{2}^{p, 0}$ by Lemma 4.7.

Proof. We first claim that $\phi^{*}: \mathrm{H}_{\text {ét }}^{p}\left(X_{\mathrm{a}}, \mathbb{Q}_{\ell}\right) \rightarrow \mathrm{H}_{\text {ét }}^{p}\left(\mathcal{X}_{\eta, \mathrm{a}}, \mathbb{Q}_{\ell}\right)$ is injective. In fact, for every $\alpha \in$ $\mathrm{H}_{\text {ét }}^{p}\left(X_{\mathrm{a}}, \mathbb{Q}_{\ell}\right)$ and $\beta \in \mathrm{H}_{\text {ét }}^{2 \operatorname{dim} X-p}\left(X_{\mathrm{a}}, \mathbb{Q}_{\ell}\right)$, we have $\phi^{*} \alpha \cup \phi^{*} \beta=\phi^{*}(\alpha \cup \beta)$. If $\phi^{*} \alpha=0$, then $\phi^{*}(\alpha \cup \beta)=0$. However, since $\phi^{*}: \mathrm{H}_{\text {ét }}^{2 \operatorname{dim} X}\left(X_{\mathrm{a}}, \mathbb{Q}_{\ell}\right) \rightarrow \mathrm{H}_{\text {ét }}^{2 \operatorname{dim} X}\left(\mathcal{X}_{\eta, \mathrm{a}}, \mathbb{Q}_{\ell}\right)$ is injective as $\phi$ is proper generically étale, we must have $\alpha \cup \beta=0$ for every $\beta$. Thus, $\alpha=0$ by Poincaré duality [Ber93, Theorem 7.3.1].

Now for every embedding $\iota: \mathbb{R} \hookrightarrow \mathbb{Q}_{\ell}^{\mathrm{a}}$, we have an induced map $\kappa_{\iota}: \mathrm{H}^{p}\left(X^{\mathrm{an}}, \mathbb{R}\right) \rightarrow \mathrm{E}_{2}^{p, 0} \otimes_{\mathbb{Q}_{\ell}} \mathbb{Q}_{\ell}^{\mathrm{a}}$ from the bottom map of (5.4), which is injective by the previous claim. If we can show that the diagram

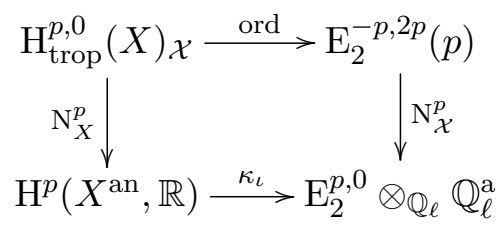

commutes for every $\iota$, then the composite map $\kappa_{\iota} \circ \mathrm{N}_{X}^{p}$ does not depend on the choice of $\iota$. An easy argument from linear algebra implies that the image of $\mathrm{N}_{X}^{p}$ must be contained in $\mathrm{H}^{p}\left(X^{\text {an }}, \mathbb{Q}\right)$ and that the original diagram (5.4) commutes.

Now we show the commutativity of (5.5). To simplify the notation, put

$$
\mathscr{A}_{?}^{p, q, \mathrm{cl}}:=\operatorname{ker}\left(\mathrm{d}^{\prime \prime}: \mathscr{A}_{?}^{p, q} \rightarrow \mathscr{A}_{?}^{p, q+1}\right) .
$$

Take an element $\omega \in \mathrm{H}_{\text {trop }}^{p, 0}(X) \mathcal{X}$. By Lemma 3.2(i), the map $\mathrm{N}_{X}^{p}(\omega)$ is represented by the Dolbeault representative $p ! \mathrm{J} \omega \in \mathrm{H}^{0}\left(X^{\text {an }}, \mathscr{A}_{X^{\text {an }}}^{0, p \text { cl }}\right)$.

For $1 \leqslant i \leqslant m$, put $U^{i}:=\pi_{\mathcal{X}}^{-1} Y^{i}$, and choose a presentation $\left(U^{i},\left\{c_{l}^{i}\right\},\left\{f_{l k}^{i}\right\} \mid 1 \leqslant l \leqslant M_{i}\right.$, $1 \leqslant k \leqslant p)$ of $\omega$ on $Y^{i}$. For every subset $I \subset\{1, \ldots, m\}$, put $U^{I}:=\bigcap_{i \in I} U^{i}=\pi_{\mathcal{X}}^{-1} Y^{I}$. Since $\pi_{0}\left(U^{I}\right)=\pi_{0}\left(Y^{I}\right)$, the assignment $Z \mapsto \operatorname{ord}_{\omega}(Z)$ gives rise to a Cech $p$-cocycle $\theta_{\omega}$ for the sheaf $\mathbb{Q}$ 
with respect to the ordered open covering $\underline{U}=\left\{U^{1}, \ldots, U^{m}\right\}$ of $\mathcal{X}_{\eta}^{\text {an }}$. Since $\delta_{p}^{*} \operatorname{ord}_{\omega}=0$ by Lemma 5.9(ii), the cocycle $\theta_{\omega}$ is closed hence gives rise to a class in $\mathrm{H}^{p}(\underline{U}, \mathbb{Q})$, whose image $\left[\theta_{\omega}\right] \in \mathrm{H}^{p}\left(\mathcal{X}_{\eta}^{\text {an }}, \mathbb{Q}\right)$ coincides with $\left(\kappa_{\mathcal{X}_{\eta}}^{p}\right)^{-1}\left(\mathrm{~N}_{\mathcal{X}}^{p}\left(\operatorname{ord}_{\omega}\right)\right)$ by $(4.4)$, where $\kappa_{\mathcal{X}_{\eta}}^{p}: \mathrm{H}^{p}\left(\mathcal{X}_{\eta}^{\text {an }}, \mathbb{Q}_{\ell}\right) \stackrel{\sim}{\rightarrow} \mathrm{E}_{2}^{p, 0}$ is the isomorphism in Lemma 4.7. Therefore by Lemma 3.2(i), the commutativity of (5.5) is equivalent to the statement that $(-1)^{p(p+1) / 2} p ! \mathrm{J} \omega$ is a Dolbeault representative of $\left[\theta_{\omega}\right]$.

Let us recall the construction of Dolbeault representatives, following the similar procedure in the proof of [GH94, Section 5.1, first lemma, p. 651]. For an abelian sheaf $\mathscr{F}$ on $\mathcal{X}_{\eta}^{\text {an }}$ and $r \geqslant 0$, denote by

$$
C^{r}(\underline{U}, \mathscr{F})=\bigoplus_{|I|=r+1} \Gamma\left(U^{I}, \mathscr{F}\right)
$$

the abelian group of Čech $r$-cocycles for $\mathscr{F}$ with respect to $\underline{U}$. We have a coboundary map $\delta: C^{r}(\underline{U}, \mathscr{F}) \rightarrow C^{r+1}(\underline{U}, \mathscr{F})$. If we have elements $\theta_{r} \in C^{r}\left(\underline{U}, \mathscr{A}_{\mathcal{X}_{\eta}^{a n}}^{0, p-r-1}\right)$ for $0 \leqslant r \leqslant p-1$ satisfying

$$
\mathrm{d}^{\prime \prime} \theta_{0}=\mathrm{J} \omega, \quad \mathrm{d}^{\prime \prime} \theta_{1}=\delta \theta_{0}, \ldots, \quad \mathrm{d}^{\prime \prime} \theta_{p-1}=\delta \theta_{p-2}, \quad \frac{(-1)^{p(p+1) / 2}}{p !} \theta_{\omega}=\delta \theta_{p-1},
$$

then $(-1)^{p(p+1) / 2} p ! \mathrm{J} \omega$ is a Dolbeault representative of $\left[\theta_{\omega}\right]$ and (5.5) commutes. Here, we regard $\mathrm{J} \omega$ as an element in $C^{0}\left(\underline{U}, \mathscr{A}_{X^{\text {an }}}^{0, p}\right)$.

The remainder of the proof will be dedicated to the construction of $\theta_{r}$. For $r \geqslant 0$, denote the set of irreducible components of $Y^{[r]}$ by $\mathcal{D}^{r}$.

Step 1. For $Z \in \mathcal{D}^{r}$, put $U_{Z}:=\pi_{\mathcal{X}}^{-1} Z$ and $M_{Z}:=\sum_{i \in I(Z)} M_{i}$, and let $f_{Z}: U_{Z} \rightarrow\left(\mathbf{G}_{m}^{\text {an }}\right)^{M_{Z} p}$ be the moment map given by invertible functions $\left\{f_{l k}^{i} \mid i \in I(Z), 1 \leqslant l \leqslant M_{i}, 1 \leqslant k \leqslant p\right\}$ on $U$. Put $U_{Z}^{\circ}:=\pi_{\mathcal{X}}^{-1}\left(Z \backslash Y^{[r+1]}\right)$. The image of the tropicalization map $f_{Z \text {,trop }}: U_{Z} \rightarrow \mathbb{R}^{M_{Z} p}$, denoted by $C_{Z}$, is canonically a simplicial complex, induced by the reduced, normal crossing divisor $Z \cap Y^{[r+1]}$ of $Z$, with the unique minimal simplex $\Delta_{Z}:=f_{Z \text {,trop }}\left(U_{Z}^{\circ}\right)$. We now define the barycenter $P_{Z}$ of $\Delta_{Z}$ as follows.

Let $\eta_{Z}$ be the generic point of $Z$. Let $\left(\mathcal{V},\left\{g_{0}, \ldots, g_{r}\right\}\right)$ be a semistable chart at $\eta_{Z}$ (Definition 4.3). We let $g_{Z}: U_{Z} \cap \mathcal{V}_{\eta}^{\text {an }} \rightarrow\left(\mathbf{G}_{m}^{\text {an }}\right)^{r+1}$ be the moment map induced by $\left\{g_{0}, \ldots, g_{r}\right\}$. Then the image of $g_{Z \text {,trop }}$ is the standard (open) simplex $\Delta^{\circ}$ in $\mathbb{R}^{r+1}$, which is $\left\{\left(x_{0}, \ldots, x_{r}\right) \in \mathbb{R}^{r+1} \mid\right.$ $\left.x_{0}+\cdots+x_{r}=1, x_{i}>0\right\}$. As in the proof of Lemma 5.9, we have unique integers $a_{l k j}^{i}$ such that

$$
f_{l k}^{i} \cdot \prod_{j=0, j \neq i}^{r} g_{j}^{-a_{l k j}^{i}}
$$

has norm 1 on $\pi_{\mathcal{X}}^{-1}(Z \cap \mathcal{V}) \subset U_{Z}^{\circ}$. Then there exists a unique homomorphism

$$
h_{Z}:\left(\mathbf{G}_{m}^{\text {an }}\right)^{r+1} \rightarrow\left(\mathbf{G}_{m}^{\text {an }}\right)^{M_{Z} p}
$$

determined by integers $\left\{a_{l k j}^{i}\right\}$ such that $\left.f_{Z, \text { trop }}\right|_{\pi_{\mathcal{X}}^{-1}(Z \cap \mathcal{V})}=\left(h_{Z} \circ g_{Z}\right)_{\text {trop }}$. Then we have

$$
h_{Z}^{b}\left(\Delta^{\circ}\right)=f_{Z, \operatorname{trop}}\left(\pi_{\mathcal{X}}^{-1}(Z \cap \mathcal{V})\right) \subset \Delta_{Z}
$$

where $h_{Z}^{b}: \mathbb{R}^{r+1} \rightarrow \mathbb{R}^{M_{Z} p}$ is the map induced by (5.7) (Notation 5.7). We now define $P_{Z}$ to be the image of the barycenter of $\Delta^{\circ}$, which has coordinates $(1 /(r+1), \ldots, 1 /(r+1))$, under $h_{Z}^{b}$. It is easy to see that the point $P_{Z}$ does not depend on the choice of the semistable chart of $\eta_{Z}$ (and in fact, $h_{Z}^{b}\left(\Delta^{\circ}\right)=\Delta_{Z}$ ). To summarize, we have $P_{Z} \in \Delta_{Z} \subset C_{Z} \subset \mathbb{R}^{M_{Z} p}$. 


\section{MONODROMY MAP FOR TROPICAL DOLBEAUlt COHOMOLOGY}

Step 2. Take another irreducible component $Z^{\prime} \in \mathcal{D}^{r^{\prime}}$ such that $Z \subset Z^{\prime}$. In particular, we have $r^{\prime} \leqslant r$ and $I\left(Z^{\prime}\right) \subset I(Z)$. Let

$$
h_{Z, Z^{\prime}}:\left(\mathbf{G}_{m}^{\text {an }}\right)^{M_{Z} p} \rightarrow\left(\mathbf{G}_{m}^{\text {an }}\right)^{M_{Z^{\prime}} p}
$$

be the canonical projection by forgetting components with $i \in I(Z) \backslash I\left(Z^{\prime}\right)$. Then the diagram

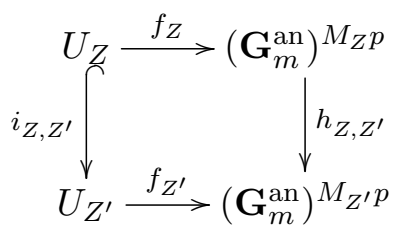

commutes, where $i_{Z, Z^{\prime}}$ is the natural inclusion map. It induces, for every $q \geqslant 0$, the commutative diagram

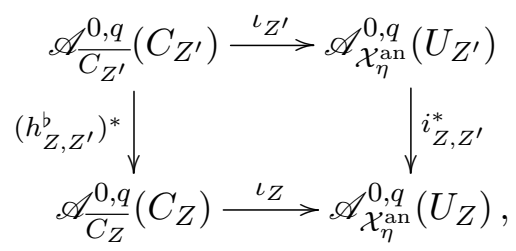

where $\iota_{Z}$ and $\iota_{Z^{\prime}}$ are natural maps from the definition of $\mathscr{A}_{\mathcal{X}_{\eta}^{\text {an }}}^{0, q}$.

On the other hand, we have a canonical isomorphism $C^{r}\left(\underline{U}, \mathscr{A}_{\mathcal{X}_{\eta}^{\text {an }}}^{0, q}\right) \simeq \bigoplus_{Z \in \mathcal{D}^{r}} \mathscr{A}_{\mathcal{X}_{\eta}^{\text {an }}}^{0, q}\left(U_{Z}\right)$, which induces a canonical map

$$
\iota^{r}: \bigoplus_{Z \in \mathcal{D}^{r}} \mathscr{A}^{0, q}\left(C_{Z}\right) \rightarrow C^{r}\left(\underline{U}, \mathscr{A}_{\mathcal{X}_{\eta}^{\text {an }}}^{0, q}\right)
$$

for $r \geqslant 0$, and there is a similarly defined coboundary map

$$
\delta: \bigoplus_{Z \in \mathcal{D}^{r}} \mathscr{A} \frac{0, q}{C_{Z}}\left(C_{Z}\right) \rightarrow \bigoplus_{Z^{\prime} \in \mathcal{D}^{r+1}} \mathscr{A} \frac{\mathscr{C}^{0, q}}{C_{Z^{\prime}}}\left(C_{Z^{\prime}}\right)
$$

using $\left(h_{Z, Z^{\prime}}^{b}\right)^{*}$ as restriction maps, that is compatible with the coboundary map for $C^{\bullet}\left(\underline{U}, \mathscr{A}_{\mathcal{X}_{\eta}^{\text {an }}}^{0, q}\right)$ under $\iota^{\bullet}$.

Moreover, since $C_{Z}$ is star-shaped with respect to $P_{Z}$, we have the star-shape integration map

$$
\mathcal{I}_{P_{Z}}^{\prime \prime}: \mathscr{A}_{C_{Z}}^{0, q}\left(C_{Z}\right) \rightarrow \mathscr{A}_{C_{Z}}^{0, q-1}\left(C_{Z}\right)
$$

for $q \geqslant 1$ (see the appendix for a formula on the standard simplex). We put

$$
\mathcal{I}^{\prime \prime}:=\bigoplus_{Z \in \mathcal{D}^{r}} \mathcal{I}_{P_{Z}}^{\prime \prime}: \bigoplus_{Z \in \mathcal{D}^{r}} \mathscr{A} \frac{{ }^{0, q}}{C_{Z}}\left(C_{Z}\right) \rightarrow \bigoplus_{Z \in \mathcal{D}^{r}} \mathscr{A}^{0, q} \bar{C}^{-1}\left(C_{Z}\right)
$$

Step 3. Note that $\mathcal{D}^{0}=\left\{Y^{i} \mid 1 \leqslant i \leqslant m\right\}$. Define $\vartheta_{0} \in \bigoplus_{Z \in \mathcal{D}^{0}} \mathscr{A}_{\bar{C}_{Z}}^{0, p-1}\left(C_{Z}\right)$ by the formula

$$
\vartheta_{0}\left(Y^{i}\right)=\mathcal{I}_{P_{Y^{i}}}^{\prime \prime}\left(\sum_{l=1}^{M_{i}} c_{l}^{i} \mathrm{~d}^{\prime \prime} x_{l 1}^{i} \wedge \cdots \wedge \mathrm{d}^{\prime \prime} x_{l p}^{i}\right),
$$

where $\left\{x_{l k}^{i}\right\}$ are the standard coordinates on $\mathbb{R}^{M_{i} p}$. For $1 \leqslant r \leqslant p-1$, define

$$
\vartheta_{r}=\mathcal{I}^{\prime \prime}\left(\delta \vartheta_{r-1}\right) \in \bigoplus_{Z \in \mathcal{D}^{r}} \mathscr{A}^{0, p-r-1}\left(C_{Z}\right)
$$


We claim that

$$
\mathrm{d}^{\prime}\left(\delta \vartheta_{r-1}\right)=\mathrm{d}^{\prime \prime}\left(\delta \vartheta_{r-1}\right)=0
$$

for $1 \leqslant r \leqslant p-1$ and

$$
\delta \vartheta_{p-1}=\frac{(-1)^{p(p+1) / 2}}{p !} \theta_{w} .
$$

We leave the proof to the next step. Assuming these, we have $\mathrm{d}^{\prime \prime} \vartheta_{r}=\delta \vartheta_{r-1}$ as $\mathrm{d}^{\prime \prime}\left(\delta \vartheta_{r-1}\right)=0$. Finally, we put $\theta_{r}=\iota^{r} \vartheta_{r} \in C^{r}\left(\underline{U}, \mathscr{A}_{\mathcal{X}_{\eta}^{\text {an }}}^{0, p-r-1}\right)$ for $0 \leqslant r \leqslant p-1$. The $\theta_{r}$ satisfy (5.6), and the lemma follows.

Step 4. We have to verify (5.11) and (5.12) for $\left(\delta \vartheta_{r-1}\right)(Z) \in \mathscr{A}_{C_{Z}}^{0, p-r}\left(C_{Z}\right)$ for every $Z \in \mathcal{D}^{r}$. Without loss of generality, we assume that $X$ has dimension $n$. For each fixed $Z \in \mathcal{D}^{r}$, it suffices to consider the restriction of $\left(\delta \vartheta_{r-1}\right)(Z)$ to maximal (open) cells of $C_{Z}$, all of which are of the form $f_{Z \text {,trop }}\left(U_{z}\right)$, where $z \in Z$ is a closed point that belongs to $\mathcal{D}^{n}$.

The idea is to reverse the consideration. We fix a closed point $z \in \mathcal{D}^{n}$ and consider the restriction of $\left(\delta \vartheta_{r-1}\right)(Z)$ to $f_{Z \text {,trop }}\left(U_{z}\right)$ for all $1 \leqslant r \leqslant p$ and all $Z \in \mathcal{D}^{r}$ such that $z \in Z$. We fix a semistable chart $\left(\mathcal{V},\left\{g_{0}, \ldots, g_{n}\right\}\right)$ at $z$. Then $\mathcal{V}_{\eta}^{\text {an }}$ contains $U_{z}$. We have the moment map $g: \mathcal{V}_{\eta}^{\text {an }} \rightarrow\left(\mathbf{G}_{m}^{\text {an }}\right)^{n+1}$ and $g_{\text {trop }}: \mathcal{V}_{\eta}^{\text {an }} \rightarrow \mathbb{R}^{n+1}$ such that $g_{\text {trop }}\left(U_{z}\right)$ is the standard open simplex $\Delta^{\circ}$ in $\mathbb{R}^{n+1}$. Note that for every subset $I \subset I(z)$ of cardinality $r+1$, there is a unique element $Z \in \mathcal{D}^{r}$ that contains $z$, which we denote by $Z_{I}$. For every $I$ as above, put

$$
h_{I}:=h_{z, Z_{I}} \circ h_{z}:\left(\mathbf{G}_{m}^{\text {an }}\right)^{n+1} \rightarrow\left(\mathbf{G}_{m}^{\text {an }}\right)^{M_{Z_{I}} p},
$$

where $h_{z}$ and $h_{z, Z_{I}}$ are defined in (5.7) and (5.8), respectively. Then $f_{Z \text {,trop }}\left(U_{z}\right)$ is the image of $\Delta^{\circ}$ under $h_{I}^{b}$. Consider the composite map

$$
h_{I}^{\dagger}: \mathscr{A}_{\frac{0, q}{C_{Z_{I}}}}\left(C_{Z_{I}}\right) \stackrel{\left(h_{I}^{\mathrm{b}}\right)^{*}}{\longrightarrow} \mathscr{A}_{\Delta}^{0, q}\left(\Delta^{\circ}\right) \rightarrow \Omega^{q}(\Delta)
$$

where the second map is simply regarding a $(0, q)$-superform as an ordinary $q$-form (see the appendix for notation concerning $\Delta)$. Since $h_{I}^{b}$ is affine and $h_{I}\left(P^{I}\right)=P_{Z^{I}}$, the diagram

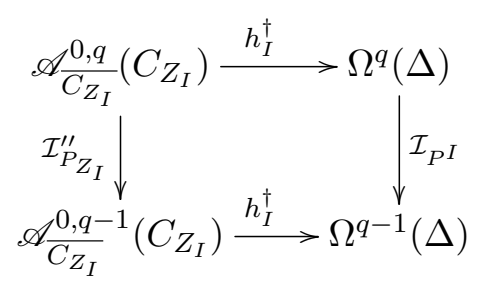

commutes. Moreover, we have the commutative diagram

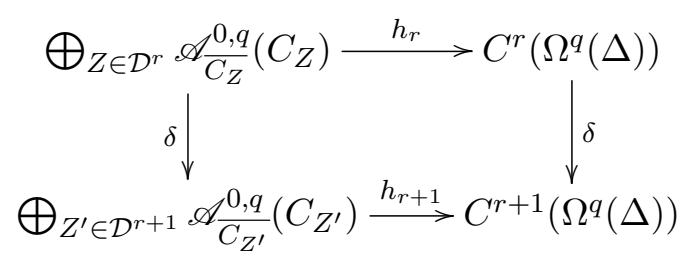

concerning coboundary maps. Here, $\delta$ on the left-hand side is (5.9) and $\delta$ on the right-hand side 


\section{Monodromy map For tropical Dolbeault COHOMOlOGY}

is defined in the appendix. The map $h_{r}$ is the composition of the projection

$$
\bigoplus_{Z \in \mathcal{D}^{r}} \mathscr{A} \frac{0, q}{C_{Z}}\left(C_{Z}\right) \rightarrow \bigoplus_{I \subset I(z),|I|=r+1} \mathscr{A} \frac{\mathscr{A}^{0, q}}{C_{Z_{I}}}\left(C_{Z_{I}}\right)
$$

and $\bigoplus_{I \subset I(z),|I|=r+1} h_{I}^{\dagger}$.

Recall that we have the element $\vartheta_{0} \in \bigoplus_{Z \in \mathcal{D}^{0}} \mathscr{A}^{0, p} \bar{C}_{Z}^{-1}\left(C_{Z}\right)$ defined in (5.10). Put $\beta_{0}:=$ $h_{0}\left(\mathrm{~d}^{\prime \prime} \vartheta_{0}\right) \in C^{0}\left(\Omega^{p}(\Delta)\right)$. Since

$$
\left(\mathrm{d}^{\prime \prime} \vartheta_{0}\right)\left(Y^{i}\right)=\sum_{l=1}^{M_{i}} c_{l}^{i} \mathrm{~d}^{\prime \prime} x_{l 1}^{i} \wedge \cdots \wedge \mathrm{d}^{\prime \prime} x_{l p}^{i}
$$

(Remark A.1), the element $h_{0}\left(\mathrm{~d}^{\prime \prime} \vartheta_{0}\right)(i)$ represents the form $\omega$ over $U_{z}$, for every $i \in I(z)$. In particular, $h_{0}\left(\mathrm{~d}^{\prime \prime} \vartheta_{0}\right)$ is of constant value $\beta \in \Omega^{p}(\Delta)$. Now we are in the situation of Proposition A.2. In particular, we have elements $\beta_{r} \in C^{r}\left(\Delta^{p-r}(\Delta)\right)$ satisfying $\beta_{r}=\delta\left(\mathcal{I} \beta_{r-1}\right)$ for $1 \leqslant r \leqslant p$. We prove by induction that

$$
\beta_{r}=h_{r}\left(\delta \vartheta_{r-1}\right)
$$

When $r=1$, we have

$$
\beta_{1}=\delta\left(\mathcal{I} \beta_{0}\right)=\delta\left(\mathcal{I}\left(h_{0}\left(\mathrm{~d}^{\prime \prime} \vartheta_{0}\right)\right)\right)=\delta\left(h_{0}\left(\mathcal{I}^{\prime \prime}\left(\mathrm{d}^{\prime \prime} \vartheta_{0}\right)\right)\right)=\delta\left(h_{0}\left(\vartheta_{0}\right)\right)=h_{1}\left(\delta \vartheta_{0}\right) .
$$

Suppose $\beta_{r}=h_{r}\left(\delta \vartheta_{r-1}\right)$. Then we have

$$
\beta_{r+1}=\delta\left(\mathcal{I} \beta_{r}\right)=\delta\left(\mathcal{I}\left(h_{r}\left(\delta \vartheta_{r-1}\right)\right)\right)=\delta\left(h_{r}\left(\mathcal{I}^{\prime \prime}\left(\delta \vartheta_{r-1}\right)\right)\right)=\delta\left(h_{r}\left(\vartheta_{r}\right)\right)=h_{r+1}\left(\delta \vartheta_{r}\right),
$$

where we recall that $\vartheta_{r}=\mathcal{I}^{\prime \prime}\left(\delta \vartheta_{r-1}\right)$ by definition.

Now Proposition A.2(i) (respectively, (ii)) implies that (5.11) (respectively, (5.12)) holds when restricted to $f_{Z \text {,trop }}\left(U_{z}\right)$. Since $z$ is arbitrary, (5.11) and (5.12) hold.

LEMma 5.11. We have $\mathrm{H}_{\text {trop }}^{p, 0}(X)=\bigcup_{\mathcal{X}} \mathrm{H}_{\text {trop }}^{p, 0}(X)_{\mathcal{X}}$, where the union is taken over all strict semistable alterations $\mathcal{X}$ of $X$.

Proof. Let $\omega$ be an element in $\mathrm{H}_{\text {trop }}^{p, 0}(X)$. Since $X$ is proper, we may choose a finite open covering $\underline{U}$ of $X^{\text {an }}$ such that for every $U \in \underline{U}$, there are $c_{l} \in \mathbb{Q}$ for $1 \leqslant l \leqslant M$ with some integer $M \geqslant 1$, and $f_{l k} \in \mathscr{O}_{X^{\text {an }}}^{*}(U)$ for $1 \leqslant l \leqslant M$ and $1 \leqslant k \leqslant p$, such that $\left.\omega\right|_{U}=\tau\left(\sum_{l=1}^{M} c_{l}\left\{f_{l 1}, \ldots, f_{l p}\right\}\right)$.

By [Liu17, Lemma 7.8], after taking a finite extension $K^{\prime} / K$ inside $K^{\text {a }}$, we have a (proper, flat) integral model $\mathcal{X}_{0}$ of $X \otimes_{K} K^{\prime}$ such that if $Y_{0}^{1}, \ldots, Y_{0}^{m_{0}}$ are all reduced irreducible components of $\mathcal{X}_{0} \otimes_{K^{\prime}} \widetilde{K^{\prime}}$, then the covering $\left\{\pi_{\mathcal{X}_{0}}^{-1} Y_{0}^{i} \mid i=1, \ldots, m_{0}\right\}$ refines $\underline{U}$. By [dJo96, Theorem 8.2], we have a proper, strictly semistable scheme $\mathcal{X}$ over $K_{\mathcal{X}}^{\circ}$, where $K_{\mathcal{X}} / K^{\prime}$ is a finite extension inside $K^{\mathrm{a}}$, with a proper, generically étale morphism $\mathcal{X} \rightarrow \mathcal{X}_{0}$. Replacing $K_{\mathcal{X}}$ by a finite unramified extension inside $K^{\text {a }}$, we may assume that every irreducible component of $Y^{(p)}$ for $p \geqslant 0$ is geometrically irreducible. Therefore, $\left(\mathcal{X}, \phi: \mathcal{X}_{\eta} \rightarrow X\right)$ is a strict semistable alteration of $X$ such that $\left\{\pi_{\mathcal{X}}^{-1} Y^{i} \mid i=\right.$ $1, \ldots, m\}$ refines $\left(\phi^{\text {an }}\right)^{-1} \underline{U}$.

Now for an arbitrary irreducible component $Y^{i}$ of $Y$, take an open subset $U \in \underline{U}$ such that $\pi_{\mathcal{X}}^{-1} Y^{i} \subset\left(\phi^{\text {an }}\right)^{-1} U$. For every $f_{l k}$ as above, we may choose an element $a_{l k} \in K^{*}$ such that $\left|a_{l k} \phi^{\text {an* }} f_{l k}\right|=1$ on $\pi_{\mathcal{X}}^{-1}\left(Y^{i} \backslash Y^{[1]}\right)$. Thus, $\left(\left(\phi^{\text {an }}\right)^{-1} U,\left\{c_{l}\right\},\left\{a_{l k} \phi^{\text {an* }} f_{l k}\right\}\right)$ is a presentation of $\omega$ on $Y^{i}$. Therefore, $\omega$ belongs to $\mathrm{H}_{\text {trop }}^{p, 0}(X) \mathcal{X}$.

Proof of Theorem 5.6. Part (i) is a consequence of Lemmas 5.10 and 5.11. 
For part (ii), it is equivalent to check that the map $\mathrm{N}_{X}^{p}: \mathrm{H}_{\text {trop }}^{p, 0}(X) \rightarrow \mathrm{H}^{p}\left(X^{\text {an }}, \mathbb{Q}\right)$ is injective. Let $\omega$ be an element of $\mathrm{H}_{\text {trop }}^{p, 0}(X)$. By Lemma 5.11, we may assume $\omega \in \mathrm{H}_{\text {trop }}^{p, 0}(X) \mathcal{X}$ for a strict semistable alteration (Definition 5.5) $\mathcal{X}$ of $X$. Then the injectivity follows from Lemmas 5.9 and 5.10 as we assume that $\mathrm{N}_{\mathcal{X}}^{p}$ is injective.

Corollary 5.12. Let $X_{0}$ be a proper smooth scheme over a non-Archimedean field $K_{0}$ that is isomorphic to $k((t))$ for $k$ either a finite field or a field of characteristic zero. Let $K$ be a closed subfield of $\widehat{K_{0}^{\mathrm{a}}}$ containing $K_{0}$. Then the monodromy map

$$
\mathrm{N}_{X}^{p}: \mathrm{H}_{\text {trop }}^{p, 0}(X)_{\mathbb{R}} \rightarrow \mathrm{H}_{\text {trop }}^{0, p}(X)_{\mathbb{R}}
$$

is injective, where $X=X_{0} \otimes_{K_{0}} K$. In particular, we have $\mathrm{h}_{\text {trop }}^{p, 0}(X)<\infty$ and $\mathrm{h}_{\text {trop }}^{p-q, q}(X) \geqslant \mathrm{h}_{\text {trop }}^{p, 0}(X)$ for $0 \leqslant q \leqslant p$.

Proof. First, suppose that $K / K_{0}$ is a finite extension. By Lemma 4.8 and resolution of singularity when char $k=0$, the map $\kappa_{X}^{p}$ is injective. Then the corollary follows from Theorem 5.6 and Proposition 4.6(ii).

In general, since $X_{0}$ is proper, we have

$$
\mathrm{H}_{\text {trop }}^{p, 0}(X)=\bigcup_{K_{0} \subset K^{\prime} \subset K} \mathrm{H}_{\text {trop }}^{p, 0}\left(X_{0} \otimes_{K_{0}} K^{\prime}\right)
$$

where the union is taken over all finite extensions $K^{\prime} / K_{0}$ contained in $K$. By [Ber99, Theorem 10.1], we know that $\mathrm{H}_{\text {trop }}^{0, p}\left(X_{0} \otimes_{K_{0}} K^{\prime}\right)$, which is isomorphic to $\mathrm{H}^{p}\left(X_{0}^{\mathrm{an}} \widehat{\otimes}_{K_{0}} K^{\prime}, \mathbb{Q}\right)$ by Remark 5.2, stabilizes when $K^{\prime}$ increases. Therefore, the injectivity of $\mathrm{N}_{X}^{p}$ is reduced to the case where $K / K_{0}$ is finite.

In the case where $p=1$, we have the following stronger result over certain non-Archimedean fields.

Theorem 5.13. Let $K_{0}$ be a local, non-Archimedean field and $X_{0}$ a proper, smooth scheme over $K_{0}$ that admits a proper, strictly semistable model over $K_{0}^{\circ}$. Then the monodromy map

$$
\mathrm{N}_{X}: \mathrm{H}_{\text {trop }}^{1,0}(X)_{\mathbb{R}} \rightarrow \mathrm{H}_{\text {trop }}^{0,1}(X)_{\mathbb{R}}
$$

is an isomorphism, where $X=X_{0} \otimes_{K_{0}} \widehat{K_{0}^{\mathrm{a}}}$.

Proof. Note that every form in $\mathrm{H}_{\text {trop }}^{1,0}(X)_{\mathbb{R}}$ and $\mathrm{H}_{\text {trop }}^{0,1}(X)_{\mathbb{R}}$ is defined over $X_{0} \otimes_{K_{0}} K$ for some finite extension $K$ of $K_{0}$ contained in $\widehat{K_{0}^{a}}$. We need some particular strict semistable alteration of $X_{0} \otimes_{K_{0}} K$ for sufficiently large $K$. Since $X_{0}$ admits a proper, strictly semistable model over $K_{0}^{\circ}$, it is well known (see, for example, [Sai03, Lemma 1.11]) that $X_{0} \otimes_{K_{0}} K$ admits a proper, strictly semistable model over $K^{\circ}$ for any finite extension of $K$ of $K_{0}$. Replacing $K$ by a finite unramified extension, we may assume that $X_{0}$ admits a strict semistable alteration $\mathcal{X}$ over $K^{\circ}$ such that $\phi: \mathcal{X}_{\eta} \rightarrow X_{0}$ induces an isomorphism $\mathcal{X}_{\eta} \stackrel{\sim}{\rightarrow} X_{0} \otimes_{K_{0}} K$. We then have an infinite sequence of successive finite field extensions $K_{0} \subset K_{1} \subset \cdots$ contained in $K_{0}^{\mathrm{a}}$ such that for $i \geqslant 1$, the scheme $X_{0}$ admits a strict semistable alteration $\mathcal{X}_{i}$ over $K_{i}^{\circ}$ with $\phi: \mathcal{X}_{i, \eta} \stackrel{\sim}{\rightarrow} X_{i}$ an isomorphism, where $X_{i}:=X_{0} \otimes_{K_{0}} K_{i}$.

By the similar argument in the proof of Corollary 5.12, it suffices to show that

$$
\mathrm{N}_{X_{i}}: \mathrm{H}_{\text {trop }}^{1,0}\left(X_{i}\right)_{\mathbb{R}} \rightarrow \mathrm{H}_{\text {trop }}^{0,1}\left(X_{i}\right)_{\mathbb{R}}
$$




\section{MONODROMY MAP FOR TROPICAL DOLBEAUlt COHOMOLOGY}

is an isomorphism for every $i \geqslant 1$. By Theorem 5.6(ii), Lemma 4.8, and Proposition 4.6(iii), we know that (5.13) is injective. Thus, if we can show $\mathrm{h}_{\text {trop }}^{1,0}\left(X_{i}\right) \geqslant \mathrm{h}_{\text {trop }}^{0,1}\left(X_{i}\right)$, then (5.13) is an isomorphism.

We fix such an index $i$ and suppress it from the notation. In particular, we now have $X=$ $X_{0} \otimes_{K_{0}} K$ with $K$ a finite extension of $K_{0}$. Let $\mathcal{X}$ be a strict semistable alteration of $X$ with an isomorphism $\phi: \mathcal{X}_{\eta} \stackrel{\sim}{\rightarrow} X$. We identify $X$ with $\mathcal{X}_{\eta}$. By Lemma 4.7 and Proposition 4.6(iii), the composite map

$$
\mathrm{N}_{\mathcal{X}}^{-1} \circ \kappa_{X}^{1}: \mathrm{H}^{1}\left(X^{\mathrm{an}}, \mathbb{Q}_{\ell}\right) \rightarrow \mathrm{E}_{2}^{-1,2}(1)
$$

is an isomorphism, under which the image of $\mathrm{H}^{1}\left(X^{\mathrm{an}}, \mathbb{Q}\right)$ is contained in $\mathrm{E}_{2}^{-1,2}(1) \cap \mathrm{H}_{\text {ét }}^{0}\left(Y_{\mathrm{s}}^{(1)}, \mathbb{Q}\right)$, which we denote by $\mathrm{E}_{\mathcal{X}}$. The theorem will follow if we can construct an injective (linear) map

$$
\mathrm{T}_{\mathcal{X}}: \mathrm{E}_{\mathcal{X}} \rightarrow \mathrm{H}^{0}\left(X^{\mathrm{an}}, \mathscr{T}_{X^{\text {an }}}^{1}\right)
$$

For every point $y \in Y$, we fix a semistable chart $\left(\mathcal{V}_{y},\left\{g_{0}^{y}, \ldots, g_{p(y)}^{y}\right\}\right)$ at $y$ (Definition 4.3). We can write an element in $\mathrm{E}_{\mathcal{X}}$ in the form

$$
D=\frac{1}{q} \sum_{Z \in \mathcal{D}^{1}} a(Z) Z
$$

for some integers $a(Z)$ and $q>0$, where the sum is taken over $\mathcal{D}^{1}$, the set of all irreducible components of $Y^{[1]}$. As $\delta_{1 *} D=0$ by the description of the map $\mathrm{N}_{\mathcal{X}}^{1}$ via (4.4), the divisor $(q D) \cap Y^{i}$ on $Y^{i}$ is cohomologically trivial for every irreducible component $Y^{i}$ of $Y(1 \leqslant i \leqslant m)$. Thus, there exists some integer $q_{i}>0$ such that $\left(q_{i} q D\right) \cap Y^{i}$ is algebraically equivalent to zero on $Y^{i}$; in particular, $\mathscr{O}_{Y^{i}}\left(\left(q_{i} q D\right) \cap Y^{i}\right)$ is an element in $\operatorname{Pic}_{Y^{i} / \widetilde{K}}^{0}(\widetilde{K})$. Since $\operatorname{Pic}_{Y^{i} / \widetilde{K}}^{0}$ is a projective scheme over the finite field $\widetilde{K}$, one may replace $q_{i}$ by some integer multiple such that $\mathscr{O}_{Y^{i}}\left(\left(q_{i} q D\right) \cap Y^{i}\right)$ is a trivial line bundle. Replacing $q$ by $q \prod_{i=1}^{m} q_{i}$, we may assume that for every $i$, there is a rational function $f_{i} \in \widetilde{K}\left(Y^{i}\right)$ such that its divisor is exactly $(q D) \cap Y^{i}$.

For every point $y$ of $Y$, we are going to construct an open neighborhood $U_{y} \subset X^{\text {an }}$ of $\pi_{\mathcal{X}}^{-1} y$ and an element $\omega_{y} \in \mathscr{T}_{X^{\text {an }}}^{1}\left(U_{y}\right)$. For every nonempty subset $I$ of $I(y)$ (Notation 4.2), let $Z_{y}^{I}$ be the unique irreducible component of $Y^{I}$ that contains $y$. Put $U_{y}:=\mathcal{V}_{y, \eta}^{\text {an }} \cap \pi_{\mathcal{X}}^{-1} Z_{y}^{I(y)}$. For $\omega_{y}$, there are two cases:

(1) If $p(y)=0$, that is, $Z_{y}^{I(y)}=Y^{i}$ for some $i$, then we set

$$
\omega_{y}=\left.\tau\left(\frac{1}{q}\left\{f_{i}^{\sharp}\right\}\right)\right|_{U_{y}},
$$

where $f_{i}^{\sharp}$ is a lift of $\left.f_{i}\right|_{\mathcal{V}_{y} \cap Y^{i}}$ to an invertible regular function on $\mathcal{V}_{y, \eta}$.

(2) If $p(y)>0$, then we set

$$
\omega_{y}=\left.\tau\left(\frac{1}{q} \prod_{j=1}^{p(y)}\left(g_{j}^{y}\right)^{a\left(Z_{y}^{\left\{i_{0}, i_{j}\right\}}\right)}\right)\right|_{U_{y}},
$$

where $I(y)=\left\{i_{0}<\cdots<i_{p(y)}\right\}$. Note that $Z_{y}^{\left\{i_{0}, i_{j}\right\}} \in \mathcal{D}^{1}$.

We claim that the $\left(U_{y}, \omega_{y}\right)$ patch together to give an element in $\mathrm{H}^{0}\left(X^{\text {an }}, \mathscr{T}_{X^{\text {an }}}^{1}\right)=\mathrm{H}_{\text {trop }}^{1,0}(X)$, which we define as $\mathrm{T}_{\mathcal{X}} D$. Given two points $y$ and $y^{\prime}$ of $Y$, this amounts to showing that $\omega_{y}=\omega_{y^{\prime}}$ on $U_{y} \cap U_{y^{\prime}}$. If one of $y$ and $y^{\prime}$ is in Case (1), then the compatibility can be shown in the same way as in the proof of Lemma 5.8. In particular, $\omega_{y}$ does not depend on the choice of the lift $f_{i}^{\sharp}$ 
in Case (1). If both $y$ and $y^{\prime}$ are in Case (2), then it is a straightforward consequence of the fact that $\delta_{1}^{*} D=0$ by the description of the map $\mathrm{N}_{\mathcal{X}}^{1}$ via (4.4). We leave the details to the reader.

By construction, $\mathrm{T}_{\mathcal{X}}$ is apparently linear and injective. The theorem is proved.

Remark 5.14. Suppose that we are in the situation of Theorem 5.13. In particular, we have a sequence of finite extensions $K_{0} \subset K_{1} \subset \cdots$ contained in $\widehat{K_{0}^{\mathrm{a}}}$ such that $\bigcup_{i \geqslant 0} K_{i}$ is an algebraic closure of $K_{0}$ and a sequence of schemes $\cdots \rightarrow \mathcal{X}_{i} \rightarrow \mathcal{X}_{i-1} \rightarrow \cdots \rightarrow \mathcal{X}_{0}$, where each $\mathcal{X}_{i}$ is a strict semistable alteration of $X_{0} \otimes_{K_{0}} K_{i}$ such that $\mathcal{X}_{i, \eta} \simeq X_{0} \otimes_{K_{0}} K_{i}$. It follows from Lemma 4.7 that there is a canonical isomorphism $\lim _{i} \mathrm{E}_{\mathcal{X}_{i}, 2}^{p, 0} \simeq \mathrm{H}_{\text {trop }}^{0, p}(X) \otimes_{\mathbb{Q}} \mathbb{Q}_{\ell}$ for $p \geqslant 0$. Thus, Conjectures 4.5 and 5.3, together with Theorem 5.6(i), imply that there should be an isomorphism $\lim _{i} \mathrm{E}_{\mathcal{X}_{i}, 2}^{-p, 2 p}(p) \simeq \mathrm{H}_{\text {trop }}^{p, 0}(X) \otimes_{\mathbb{Q}} \mathbb{Q}_{\ell}$ for $p \geqslant 0$. This is trivial when $p=0$. The case $p=1$ is essentially Theorem 5.13. For $p \geqslant 2$, we do not know how to prove this at the moment. Moreover, for general $(p, q)$, we expect some relation between $\mathrm{H}_{\text {trop }}^{p, q}(X)$ and ${\underset{\lim }{\rightarrow}}_{i} \mathrm{E}_{\mathcal{X}_{i}, 2}^{\bullet, \bullet}$ but do not know if there is one.

\section{Appendix. Star-shaped integration on simplex}

In this appendix, we prove a technical result for the local computation used in the proof of Lemma 5.10.

Let $n \geqslant 1$ be an integer. Let $\Delta \subset \mathbb{R}^{n+1}$ be the standard (closed) simplex of dimension $n$. In other words, if we denote by $x_{0}, \ldots, x_{n}$ the standard coordinates on $\mathbb{R}^{n+1}$, then

$$
\Delta=\left\{\left(x_{0}, \ldots, x_{n}\right) \mid x_{0}+\cdots+x_{n}=1, x_{i} \geqslant 0\right\} .
$$

For $p \geqslant 0$, denote by $\Omega^{p}(\Delta)$ the space of (smooth) $p$-differential forms on $\Delta$ and by $\Omega_{\mathrm{cl}}^{p}(\Delta)$ the subspace of closed forms. Let $\omega$ be an element of $\Omega^{p}(\Delta)$; then for every $0 \leqslant i \leqslant n$, the form $\omega$ can be uniquely written as

$$
\omega=\sum_{I \subset\{0, \ldots, n\} \backslash\{i\},|I|=p} f_{I}(x) \mathrm{d} x_{I},
$$

where $f_{I}$ is a continuous function on $\Delta$, smooth in the interior of $\Delta$. We say that $\omega$ has constant coefficients if $f_{I}$ is a constant function for every such $I$. Note that this property is independent of the choice of $i$. Let $\Omega_{\text {cons }}^{p}(\Delta) \subset \Omega_{\text {cl }}^{p}(\Delta)$ be the subspace of forms with constant coefficients.

For every nonempty subset $J \subset\{0, \ldots, n\}$, let $\Delta^{J}$ be the face of $\Delta$ generated by $\left\{P^{j} \mid j \in J\right\}$, where $P^{j}$ is the point with coordinates $x_{i}=\delta_{i j}$, and let $P^{J}$ be the barycenter of $\Delta^{J}$. For every point $P \in \Delta$, the simplex $\Delta$ is star-shaped with respect to $P$. Thus, one has the star-shaped integration map

$$
\mathcal{I}_{P}: \Omega^{p}(\Delta) \rightarrow \Omega^{p-1}(\Delta)
$$

for $p \geqslant 1$. We briefly recall the definition. Consider the map $\gamma_{P}: \Delta \times[0,1] \rightarrow \Delta$ sending $(x, t)$ to $(1-t) P+t x$. Then

$$
\mathcal{I}_{P}(\alpha)=\int_{0}^{1}\left\langle\frac{\partial}{\partial t}, \gamma_{P}^{*} \alpha\right\rangle \mathrm{d} t,
$$

where $\langle$,$\rangle denotes the contraction.$

Remark A.1. By the Poincaré lemma for a star-shaped region (see, for example, [Spi65, Theorem 4-11], we know that if $\mathrm{d} \alpha=0$, then $\mathrm{d}\left(\mathcal{I}_{P}(\alpha)\right)=\alpha$.

Let $F$ be an abelian group. For every $r \geqslant 0$, put

$$
C^{r}(F):=\operatorname{Map}(\{J \subset\{0, \ldots, n\}|| J \mid=r+1\}, F) .
$$




\section{MONODROMY MAP FOR TROPICAL DOLBEAUlt COHOMOLOGY}

We have a coboundary map $\delta: C^{r}(F) \rightarrow C^{r+1}(F)$ similar to the one for Cech cocycles. Namely, for $\beta \in C^{r}(F)$, we set

$$
(\delta \beta)(J)=\sum_{k=0}^{r+1}(-1)^{k} \beta\left(\left\{j_{0}, \ldots, \widehat{j_{k}}, \ldots, j_{r+1}\right\}\right)
$$

for $J=\left\{j_{0}, \ldots, j_{r+1}\right\}$ with $0 \leqslant j_{0}<\cdots<j_{r+1} \leqslant n$.

For $p-r \geqslant 1$, we define the map

$$
\mathcal{I}: C^{r}\left(\Omega^{p-r}(\Delta)\right) \rightarrow C^{r}\left(\Omega^{p-r-1}(\Delta)\right)
$$

sending $\omega_{r}$ in the source to $\mathcal{I} \omega_{r}$ in the target such that $\left(\mathcal{I} \omega_{r}\right)(J)=\mathcal{I}_{P^{J}}\left(\omega_{r}(J)\right)$.

Proposition A.2. Let $1 \leqslant p \leqslant n$ be an integer. Let $\beta$ be an element in $\Omega_{\text {cons }}^{p}(\Delta)$. We define $\beta_{0} \in C^{0}\left(\Omega^{p}(\Delta)\right)$ by $\beta_{0}(\{j\})=\beta$ for every $0 \leqslant j \leqslant n$. For $1 \leqslant r \leqslant p$, we inductively define $\beta_{r} \in C^{r}\left(\Omega^{p-r}(\Delta)\right)$ by the formula $\beta_{r}=\delta\left(\mathcal{I} \beta_{r-1}\right)$. Then

(i) $\beta_{r}$ belongs to $C^{r}\left(\Omega_{\text {cons }}^{p-r}(\Delta)\right)$ for $0 \leqslant r \leqslant p$, and in particular $\beta_{p} \in C^{p}(\mathbb{R})$; and

(ii) we have the formula

$$
\left.\beta\right|_{\Delta^{I}}=(-1)^{p(p+1) / 2} p ! \cdot \beta_{p}(I) \mathrm{d} x_{i_{1}} \wedge \cdots \wedge \mathrm{d} x_{i_{p}}
$$

for every subset $I=\left\{i_{0}<\cdots<i_{p}\right\} \subset\{1, \ldots, n\}$.

Proof. We fix some $i \in\{0, \ldots, n\}$ and write

$$
\beta=\sum_{I \subset\{0, \ldots, n\} \backslash\{i\},|I|=p} f_{I} \mathrm{~d} x_{I}
$$

for some $f_{I} \in \mathbb{R}$. Regard $\beta$ as a $p$-form on $\mathbb{R}^{n+1}$ with constant coefficients, which we denote by $\beta^{\prime} \in \Omega_{\text {cons }}^{p}\left(\mathbb{R}^{n+1}\right)$. For a point $Q \in \mathbb{R}^{n+1}$ and $r \geqslant 1$, denote by

$$
\mathcal{I}_{Q}^{\prime}: \Omega^{r}\left(\mathbb{R}^{n+1}\right) \rightarrow \Omega^{r-1}\left(\mathbb{R}^{n+1}\right)
$$

the corresponding star-shaped integral on $\mathbb{R}^{n+1}$ with respect to $Q$. If $Q$ belongs to $\Delta$, then for $\alpha \in \Omega^{r}\left(\mathbb{R}^{n+1}\right)$, we have

$$
\left.\mathcal{I}_{Q}^{\prime}(\alpha)\right|_{\Delta}=\mathcal{I}_{Q}\left(\left.\alpha\right|_{\Delta}\right) .
$$

Suppose $Q=\left(q_{0}, \ldots, q_{n}\right)$; we define a map $\mathcal{C}_{Q}: \Omega_{\text {cons }}^{r}\left(\mathbb{R}^{n+1}\right) \rightarrow \Omega_{\text {cons }}^{r-1}\left(\mathbb{R}^{n+1}\right)$ sending $\alpha$ to the contraction of $\alpha$ by $\sum_{j=0}^{n} q_{j}\left(\partial / \partial x_{j}\right)$ (from the left). For example,

$$
\mathcal{C}_{P^{j}}\left(\mathrm{~d} x_{0} \wedge \cdots \wedge \mathrm{d} x_{r-1}\right)= \begin{cases}(-1)^{j} \mathrm{~d} x_{0} \wedge \cdots \wedge \widehat{\mathrm{d} x_{j}} \wedge \cdots \wedge \mathrm{d} x_{r-1}, & 0 \leqslant j \leqslant r-1, \\ 0, & r \leqslant j \leqslant n .\end{cases}
$$

Denote the origin of $\mathbb{R}^{n+1}$ by $O$. It is an elementary exercise to see that for $\alpha \in \Omega_{\text {cons }}^{r}\left(\mathbb{R}^{n+1}\right)$, we have

$$
\mathcal{I}_{Q}^{\prime}(\alpha)-\mathcal{I}_{O}^{\prime}(\alpha)=-\frac{1}{r} \mathcal{C}_{Q}(\alpha)
$$

For $0 \leqslant r \leqslant p$, we define an element $\beta_{r}^{\prime} \in C^{r}\left(\Omega_{\text {cons }}^{p-r}\left(\mathbb{R}^{n+1}\right)\right)$ by the formula

$$
\beta_{r}^{\prime}(I)=\frac{(-1)^{r}}{p(p-1) \cdots(p-r+1)} \sum_{j=0}^{r}(-1)^{j}\left(\mathcal{C}_{P^{i_{0}}} \circ \cdots \circ \widehat{\mathcal{C}_{P^{i_{j}}}} \circ \cdots \circ \mathcal{C}_{P^{i_{r}}}\right)\left(\beta^{\prime}\right) .
$$


for $I=\left\{i_{0}<\cdots<i_{r}\right\}$. We claim that

$$
\beta_{r}^{\prime}=\delta\left(\mathcal{I}^{\prime} \beta_{r-1}^{\prime}\right),
$$

where $\mathcal{I}^{\prime}: C^{r}\left(\Omega^{p-r}\left(\mathbb{R}^{n+1}\right)\right) \rightarrow C^{r}\left(\Omega^{p-r-1}\left(\mathbb{R}^{n+1}\right)\right)$ is defined similarly to (A.1). In fact, by the definition of $\delta$, for $r \geqslant 1$, we have

$$
\delta\left(\mathcal{I}^{\prime} \beta_{r-1}^{\prime}\right)=\sum_{j=0}^{r}(-1)^{j} \mathcal{I}_{P^{I \backslash\left\{i_{j}\right\}}}^{\prime}\left(\beta_{r-1}^{\prime}\left(I \backslash\left\{i_{j}\right\}\right)\right) .
$$

As $\beta_{r-1}^{\prime}$ is a closed cocycle, we have $\sum_{j=0}^{r}(-1)^{j} \beta_{r-1}^{\prime}\left(I \backslash\left\{i_{j}\right\}\right)=0$. Thus,

$$
\begin{aligned}
(\mathrm{A} .7) & =\sum_{j=0}^{r}(-1)^{j}\left(\mathcal{I}_{P^{I \backslash\left\{i_{j}\right\}}}^{\prime}-\mathcal{I}_{O}^{\prime}\right)\left(\beta_{r-1}^{\prime}\left(I \backslash\left\{i_{j}\right\}\right)\right) \\
& =\frac{-1}{p-r+1} \sum_{j=0}^{r}(-1)^{j} \mathcal{C}_{P^{I \backslash\left\{i_{j}\right\}}}\left(\beta_{r-1}^{\prime}\left(I \backslash\left\{i_{j}\right\}\right)\right)
\end{aligned}
$$

by (A.4), which equals

$$
\frac{(-1)^{r}}{p(p-1) \cdots(p-r+1)} \sum_{j=0}^{r}(-1)^{j} \mathcal{C}_{\left.P^{I \backslash\{i j}\right\}}\left(\sum_{k=0}^{r} \epsilon(j, k) \mathcal{C}(j, k)\left(\beta^{\prime}\right)\right) .
$$

Here $\epsilon(j, k)$ is set to be $(-1)^{k}$ (respectively, $0,(-1)^{k+1}$ ) if $k<j$ (respectively, $k=j, k>j$ ), and $\mathcal{C}(j, k)$ is the map $\mathcal{C}_{P^{i_{0}}} \circ \cdots \circ \mathcal{C}_{P^{i_{r}}}$ with $\mathcal{C}_{P^{i_{j}}}$ and $\mathcal{C}_{P^{i_{k}}}$ removed. Since

$$
\mathcal{C}_{P^{I \backslash\left\{i_{j}\right\}}}=\frac{1}{r}\left(\mathcal{C}_{P^{i_{0}}}+\cdots+\widehat{\mathcal{C}_{P^{i_{j}}}}+\cdots+\mathcal{C}_{P^{i_{r}}}\right),
$$

it is easy to see that

$$
(\mathrm{A} .8)=\frac{(-1)^{r}}{p(p-1) \cdots(p-r+1)} \sum_{j=0}^{r}(-1)^{j} \mathcal{C}(j, j)(\beta)=\beta_{r}^{\prime}(I) .
$$

Thus, (A.6) holds. By (A.3), we have $\beta_{r}=\left.\beta_{r}^{\prime}\right|_{\Delta}$ which belongs to $C^{r}\left(\Omega_{\mathrm{cons}}^{p-r}(\Delta)\right)$. Thus, statement (i) follows.

Now we compute the value of $\beta_{p}(I)=\beta_{p}^{\prime}(I)$ for $I=\left\{i_{0}<\cdots<i_{p}\right\}$. Note that the representative $\beta$ defined in (A.2) can be chosen with respect to an arbitrary $i \in\{0, \ldots, n\}$. In particular, we may take $i=i_{0}$. By (A.5), we have

$$
\beta_{p}(I)=\frac{(-1)^{p}}{p !}\left(\mathcal{C}_{P^{i_{1}}} \circ \cdots \circ \mathcal{C}_{P^{i_{p}}}\right)(\beta)=\frac{(-1)^{p(p+1) / 2}}{p !}\left(\mathcal{C}_{P^{i_{p}}} \circ \cdots \circ \mathcal{C}_{P^{i_{1}}}\right)(\beta) .
$$

Thus, statement (ii) follows as $\left.\beta\right|_{\Delta^{I}}=\left(\mathcal{C}_{P^{i_{p}}} \circ \cdots \circ \mathcal{C}_{P^{i_{1}}}\right)(\beta) \mathrm{d} x_{i_{1}} \wedge \cdots \wedge \mathrm{d} x_{i_{p}}$.

\section{ACKNOWLEDGEMENTS}

The author would like to thank Walter Gubler, Philipp Jell, and Klaus Künnemann for helpful discussions and their hospitality during his visit at Universität Regensburg. He would also like to thank Erwan Brugallé and Philipp Jell for drawing his attention to the work of Mikhalkin and Zharkov [MZ14], and the anonymous referee for very careful reading and valuable comments. 


\section{Monodromy MAP FOR TROPiCAL DOLBEAUlt COHOMOLOGY}

\section{REFERENCES}

SGA4-2 M. Artin, A. Grothendieck, and J.-L. Verdier, Théorie des topos et cohomologie étale des schémas. Séminaire de Géométrie Algébrique du Bois-Marie 1963-1964 (SGA 4). Tome 2, Lecture Notes in Math., vol. 270 (Springer-Verlag, Berlin - Heidelberg, 1972); doi:10.1007/ BFb0061319.

Ber93 V. G. Berkovich, Étale cohomology for non-Archimedean analytic spaces, Publ. Math. Inst. Hautes Études Sci. 78 (1993), no. 1, 5-161; doi:10.1007/BF02712916.

Ber99 Smooth p-adic analytic spaces are locally contractible, Invent. Math. 137 (1999), no. 1, 1-84; doi:10.1007/s002220050323.

Ber00 _ An analog of Tate's conjecture over local and finitely generated fields, Int. Math. Res. Not. 2000 (2000), no. 13, 665-680; doi:10.1155/S1073792800000362.

BGS95 S. Bloch, H. Gillet, and C. Soulé, Non-Archimedean Arakelov theory, J. Algebraic Geom. 4 (1995), no. 3, 427-485.

CD12 A. Chambert-Loir and A. Ducros, Formes différentielles réalles et courants sur les espaces de Berkovich, 2012, arXiv:1204.6277.

GH94 P. Griffiths and J. Harris, Principles of algebraic geometry (John Wiley \& Sons, Inc., New York, 1994); doi:10.1002/9781118032527.

IKMZ16 I. Itenberg, L. Katzarkov, G. Mikhalkin, and I. Zharkov, Tropical homology, 2016, arXiv:1604. 01838.

Ito05 T. Ito, Weight-monodromy conjecture over equal characteristic local fields, Amer. J. Math. 127 (2005), no. 3, 647-658; doi:10.1353/ajm.2005.0022.

Jel16 P. Jell, Real-valued differential forms on Berkovich analytic spaces and their cohomology, Ph.D. Thesis, Universität Regensburg, 2016, available at https://epub.uni-regensburg.de/ 34788/1/Thesis Jell.pdf.

dJo96 A. J. de Jong, Smoothness, semi-stability and alterations, Publ. Math. Inst. Hautes Études Sci. 83 (1996), no. 1, 51-93; doi:10.1007/BF02698644.

JSS18 P. Jell, K. Shaw, and J. Smacka, Superforms, tropical cohomology, and Poincaré duality, Adv. Geom. 19 (2019), no. 1, 101-130; doi:10.1515/ADVGEOM.2018.0006.

JW18 P. Jell and V. Wanner, Poincaré duality for the tropical Dolbeault cohomology of nonarchimedean Mumford curves, J. Number Theory 187 (2018), 344-371; doi:10.1016/ j.jnt.2017.11.004.

Liu17 Y. Liu, Tropical cycle classes for non-Archimedean spaces and weight decomposition of de Rham cohomology sheaves, Ann. Sci. Éc. Norm. Supér., to appear, available at https://gauss . math. yale.edu/ yl2269/deRham.pdf.

MZ14 G. Mikhalkin and I. Zharkov, Tropical eigenwave and intermediate Jacobians, in Homological mirror symmetry and tropical geometry, Lect. Notes Unione Mat. Ital., vol. 15 (Springer, Cham, 2014), 309-349; doi:10.1007/978-3-319-06514-4_7.

RZ82 M. Rapoport and T. Zink, Über die lokale Zetafunktion von Shimuravarietäten. Monodromiefiltration und verschwindende Zyklen in ungleicher Charakteristik, Invent. Math. 68 (1982), no. 1, 21-101; doi:10.1007/BF01394268.

Sai03 T. Saito, Weight spectral sequences and independence of l, J. Inst. Math. Jussieu 2 (2003), no. 4, 583-634; doi:10.1017/S1474748003000173.

Spi65 M. Spivak, Calculus on manifolds. A modern approach to classical theorems of advanced calculus (W. A. Benjamin, Inc., New York - Amsterdam, 1965).

Yifeng Liu yifeng.liu@yale.edu

Department of Mathematics, Northwestern University, Evanston, IL 60208, USA

Current address: Department of Mathematics, Yale University, New Haven, CT 06511, USA 